\title{
Synthesis of the ABCD Ring of Gambierol
}

Hiroki Furuta, ${ }^{\dagger}$ Mariko Hase,${ }^{\dagger}$ Ryoji Noyori, ${ }^{\ddagger}$ and Yuji Mori ${ }^{*}{ }^{\dagger}$

${ }^{\dagger}$ Faculty of Pharmacy, Meijo University, 150 Yagotoyama, Tempaku, Nagoya 468-8503, Japan ${ }^{\ddagger}$ Department of Chemistry and Research Center for Materials Science, Nagoya University, Chikusa, Nagoya 464-8606, Japan

\section{SUPPORTING INFORMATION}

Table of Contents

\begin{tabular}{|c|c|c|c|}
\hline \multicolumn{4}{|c|}{ Experimental } \\
\hline General & S-2 & Compound 12 & S-5 \\
\hline Compound 5 & S-2 & Compound 13 & S-5 \\
\hline Compound 6 & S-2 & Compound 14 & S-6 \\
\hline Compound 7 & S-3 & Compound 15 & S-6 \\
\hline Compound 8 & $S-3$ & Compound 18 & S-6 \\
\hline Compound 9 & $\mathrm{~S}-3$ & Compound 19 & S-7 \\
\hline Compound $\mathbf{1 0}$ & S-4 & Compound $\mathbf{2 0}$ & S-8 \\
\hline Compound 11 & S-4 & Compound 22 & S-8 \\
\hline \multicolumn{4}{|c|}{${ }^{1} \mathrm{H}$ and ${ }^{13} \mathrm{C}$ NMR spectra } \\
\hline Compound 5 & S-10 & Compound 13 & S-16 \\
\hline Compound $7\left({ }^{1} \mathrm{H}\right)$ & S-11 & Compound 14 & S-17 \\
\hline Compound $8\left({ }^{1} \mathrm{H}\right)$ & S-11 & Compound 15 & $\mathrm{~S}-28$ \\
\hline Compound 9 & $\mathrm{~S}-12$ & Compound $\mathbf{1 8}$ & S-19 \\
\hline Compound $\mathbf{1 0}$ & S-13 & Compound 19 & $\mathrm{~S}-20$ \\
\hline Compound 11 & S-14 & Compound $\mathbf{2 0}$ & $\mathrm{S}-21$ \\
\hline Compound 12 & S-15 & Compound 22 & $\mathrm{~S}-22$ \\
\hline
\end{tabular}


General: IR spectra were recorded in $\mathrm{CHCl}_{3}$ solution on a JASCO FTIR-420 spectrometer. ${ }^{1} \mathrm{H}$ and ${ }^{13} \mathrm{C}$ NMR spectra were recorded on a JEOL A-400 or A-600 spectrometer in $\mathrm{CDCl}_{3}$ solution using TMS and $\mathrm{CDCl}_{3}(77.00 \mathrm{ppm})$ as internal standards, respectively. EI and FAB mass spectra were obtained on JEOL JMS-700 and HX-110 mass spectrometers, respectively. Optical rotations were determined on a JASCO DIP-370 digital polarimeter. All air- and moisture-sensitive reactions were carried out under an argon atmosphere in dry, freshly distilled solvents under anhydrous conditions. Flash chromatography was carried out with E. Merck silica gel 60 (230-400 mesh). The term "dried" refers to the drying of an organic solution over $\mathrm{MgSO}_{4}$ followed by filtration.

\section{Compound 5}

To a stirred solution of diol $4(1.02 \mathrm{~g}, 3.21 \mathrm{mmol})$ in $\mathrm{CH}_{2} \mathrm{Cl}_{2}(16 \mathrm{~mL})$ and 2,6-lutidine $(1.90 \mathrm{~mL}, 16.33 \mathrm{mmol})$ at $-78^{\circ} \mathrm{C}$ was added $\mathrm{Tf}_{2} \mathrm{O}(0.57 \mathrm{~mL}, 3.36 \mathrm{mmol})$. After stirring at $-78{ }^{\circ} \mathrm{C}$ for $30 \mathrm{~min}$, TESOTf $(1.10 \mathrm{~mL}, 4.90 \mathrm{mmol})$ was added and stirring was continued for another $30 \mathrm{~min}$. The reaction was quenched with saturated aqueous $\mathrm{NaHCO}_{3}$ and the mixture was extracted with EtOAc. The extract was washed with water and brine, dried, and concentrated in vacuo. Purification by flash chromatography (3\% EtOAc in hexane) gave triflate $5(1.74 \mathrm{~g}, 94 \%)$ as a pale yellow oil. [ $\alpha]^{25}$ -37.6 (c 1.27, $\left.\mathrm{CHCl}_{3}\right)$; IR $\left(\mathrm{CHCl}_{3}\right)$ 1473, 1415, 1245, 1145, 1096, $\mathrm{cm}^{-1} ;{ }^{1} \mathrm{H} \mathrm{NMR}\left(400 \mathrm{MHz}, \mathrm{CDCl}_{3}\right) \delta 0.60-0.66(6 \mathrm{H}$, m), $0.97(9 \mathrm{H}, \mathrm{t}), 0.99(9 \mathrm{H}, \mathrm{s}), 1.04(9 \mathrm{H}, \mathrm{s}), 1.55(1 \mathrm{H}, \mathrm{dd}, J=11.7,11.2 \mathrm{~Hz}), 2.44(1 \mathrm{H}, \mathrm{ddd}, J=12.2,12.2,4.4 \mathrm{~Hz}), 3.32$ $(1 \mathrm{H}, \mathrm{ddd}, J=9.8,9.3,4.9 \mathrm{~Hz}), 3.44(1 \mathrm{H}, \mathrm{ddd}, J=9.3,5.4,1.9 \mathrm{~Hz}), 3.62(1 \mathrm{H}, \mathrm{ddd}, J=14.1,9.3,4.9 \mathrm{~Hz}), 3.75(1 \mathrm{H}, \mathrm{ddd}$, $J=13.7,6.8,4.4 \mathrm{~Hz}), 3.78(1 \mathrm{H}, \mathrm{t}, J=10.3 \mathrm{~Hz}), 4.14(1 \mathrm{H}, \mathrm{dd}, J=10.3,4.9 \mathrm{~Hz}), 4.52(1 \mathrm{H}, \mathrm{dd}, J=10.3,5.4 \mathrm{~Hz}), 4.70$ $(1 \mathrm{H}, \mathrm{dd}, J=10.7,1.9 \mathrm{~Hz}) ;{ }^{13} \mathrm{C} \mathrm{NMR}\left(100 \mathrm{MHz}, \mathrm{CDCl}_{3}\right) \delta 4.93(3 \times \mathrm{C}), 6.72(3 \mathrm{xC}), 19.9,22.6,27.03$ (3xC), $27.04(3 \times \mathrm{C})$, $42.1,65.7,66.5,71.6,75.1,77.1,79.3$. The carbon signal assigned to $\mathrm{CF}_{3}$ was not observed due to the large $J_{\mathrm{C}-\mathrm{F}}$ value. FABMS $m / z 565\left(\mathrm{MH}^{+}\right)$; HREIMS $m / z$ calcd for $\mathrm{C}_{22} \mathrm{H}_{44} \mathrm{~F}_{3} \mathrm{O}_{7} \mathrm{SSi}_{2}$ 565,2298, found 564.2231.

\section{Compound 6}

To a solution of $d l$-chloromethyl phenyl sulfoxide $(1.48 \mathrm{~g}, 8.49 \mathrm{mmol})$ in THF $(35 \mathrm{~mL})$ at $-78{ }^{\circ} \mathrm{C}$ was added LiHMDS (8.0 mL of a $1.06 \mathrm{M}$ solution in hexane) and the reaction mixture was stirred for $30 \mathrm{~min}$. A solution of (E)-4-[(4-methoxybenzyloxy)methyl]pent-3-enal $(0.99 \mathrm{~g}, 4.24 \mathrm{mmol})$ in THF $(7 \mathrm{~mL})$ was added to the solution and the reaction mixture was stirred at $-78^{\circ} \mathrm{C}$ for $1.5 \mathrm{~h}$. The reaction was quenched with saturated aqueous $\mathrm{NH}_{4} \mathrm{Cl}$ and the mixture was extracted with EtOAc. The extract was washed with water and brine, dried, and concentrated in vacuo. Purification by flash chromatography (50\% EtOAc in hexane) gave a diastereoisomeric mixture of chlorohydrins (1.37g, $79 \%$ ) as a colorless oil.

To a solution of the above chlorohydrins $(1.37 \mathrm{~g}, 3.37 \mathrm{mmol})$ in $\mathrm{CH}_{2} \mathrm{Cl}_{2}(22 \mathrm{~mL})$ and $t$ - $\mathrm{BuOH}(11 \mathrm{~mL})$ at $0^{\circ} \mathrm{C}$ was added $t$-BuOK $(416 \mathrm{mg}, 3.70 \mathrm{mmol}$ ), and the reaction mixture was stirred at room temperature for $1.5 \mathrm{~h}$. The reaction was quenched with saturated aqueous $\mathrm{NH}_{4} \mathrm{Cl}$ and the mixture was extracted with $\mathrm{Et}_{2} \mathrm{O}$. The extract was washed with water and brine, dried, and concentrated in vacuo. Purification by flash chromatography (40\% EtOAc in hexane) gave a diastereoisomeric mixture of epoxy sulfoxides $(1.04 \mathrm{~g}, 82 \%)$ as a colorless oil. The ${ }^{1} \mathrm{H}$ NMR analysis revealed that the mixture is a 1: 4 mixture of cis- and trans-epoxy sulfoxides.

To a solution of the epoxy sulfoxides $(1.04 \mathrm{~g}, 2.79 \mathrm{mmol})$ in $\mathrm{MeOH}(28 \mathrm{~mL})$ at $0{ }^{\circ} \mathrm{C}$ was added magnesium monoperoxyphthalate hexahydrate $(2.07 \mathrm{~g}, 4.18 \mathrm{mmol})$ and the reaction mixture was stirred at $0^{\circ} \mathrm{C}$ for $2.5 \mathrm{~h}$. The reaction was quenched with saturated aqueous $\mathrm{Na}_{2} \mathrm{~S}_{2} \mathrm{O}_{3}$ and the mixture was extracted with EtOAc. The extract was washed with saturated aqueous $\mathrm{NaHCO}_{3}$ and brine, dried, and concentrated in vacuo. Purification by flash 
chromatography (25\% EtOAc in hexane) gave trans-epoxy sulfone $6(750 \mathrm{mg}, 69 \%)$ as a colorless oil and cis-epoxy sulfoxide (214 mg, 21\%) was recovered. IR $\left(\mathrm{CHCl}_{3}\right)$ 1612, 1514, 1448, 1327, 1249, 1155, $1087 \mathrm{~cm}^{-1}$; ${ }^{1} \mathrm{H}$ NMR (400 $\left.\mathrm{MHz}, \mathrm{CDCl}_{3}\right) \delta 1.65(3 \mathrm{H}, \mathrm{s}), 2.51(2 \mathrm{H}, \mathrm{m}), 3.27(1 \mathrm{H}, \mathrm{ddd}, J=5.4,5.4,2,0 \mathrm{~Hz}), 3.81(3 \mathrm{H}, \mathrm{s}), 3.86(2 \mathrm{H}, \mathrm{s}), 3.93(1 \mathrm{H}, \mathrm{d}, J$ $=2.0 \mathrm{~Hz}), 4.38(2 \mathrm{H}, \mathrm{s}), 5.38(1 \mathrm{H}$, br t,$J=7.3 \mathrm{~Hz}), 6.88$ and 7.24 (each $2 \mathrm{H}, \mathrm{d}, J=8.8 \mathrm{~Hz}), 7.59(2 \mathrm{H}, \mathrm{m}), 7.70(1 \mathrm{H}, \mathrm{m})$, $7.90(2 \mathrm{H}, \mathrm{m}) ;{ }^{13} \mathrm{C}$ NMR $\left(100 \mathrm{MHz}, \mathrm{CDCl}_{3}\right) \delta 14.2,28,2,55.3,56.9,67.7,71.6,75.0,113.8,118.5,128.3,128.7$ (2xC), 129.3, $129.4(2 \times C), 130.3,134.4(2 \times C), 137.5$ (2xC), 159.2; EIMS m/z $388\left(\mathrm{M}^{+}\right)$; HREIMS $\mathrm{m} / \mathrm{z}$ calcd for $\mathrm{C}_{21} \mathrm{H}_{24} \mathrm{O}_{5} \mathrm{~S}$ $\left(\mathrm{M}^{+}\right)$388.1343, found 388.1367 .

\section{Compound 7}

A solution of triflate $5(1.61 \mathrm{~g}, 2.85 \mathrm{mmol})$ and epoxy sulfone $\mathbf{6}(1.44 \mathrm{~g}, 3.71 \mathrm{mmol})$ in HMPA (2.14 $\mathrm{mL}, 12.280 \mathrm{mmol})$ and THF (41 mL) was cooled to $-100^{\circ} \mathrm{C}$, and $n$-BuLi (2.33 mL of a $1.59 \mathrm{M}$ solution in hexane, $3.70 \mathrm{mmol}$ ) was added dropwise. After stirring at $-100{ }^{\circ} \mathrm{C}$ for $30 \mathrm{~min}$, the reaction was quenched with saturated aqueous $\mathrm{NH}_{4} \mathrm{Cl}$. The reaction mixture was warmed to room temperature and extracted with EtOAc. The extract was washed with water and brine, dried, and concentrated in vacuo. Purification by flash chromatography (20\% EtOAc in hexane) gave 7 (2.04 g, $89 \%$ as a 1:1 mixture of diastereoisomers) as a colorless oil. FABMS $m / z 803\left(\mathrm{MH}^{+}\right)$.

\section{Compound 8}

(i) Desilylation. A solution of $7(2.04 \mathrm{~g}, 2.54 \mathrm{mmol})$ and $\mathrm{TsOH} \cdot \mathrm{H}_{2} \mathrm{O}(24 \mathrm{mg}, 0.127 \mathrm{mmol})$ in $\mathrm{MeOH}(23 \mathrm{~mL})$ and $\mathrm{CH}_{2} \mathrm{Cl}_{2}(2 \mathrm{~mL})$ was stirred at room temperature for $1 \mathrm{~h}$. The reaction was quenched with $\mathrm{Et}_{3} \mathrm{~N}(0.2 \mathrm{~mL})$ and the reaction mixture was concentrated in vacuo. The residue was purified by flash chromatography (30\% EtOAc in hexane) to give a 1:1 mixture of hydroxy epoxy sulfones $(1.73 \mathrm{~g}, 99 \%)$ as a colorless oil.

(ii) Bromo ketone formation. To a stirred solution of the hydroxy epoxy sulfone (1.04 g, 1.52 mmol) and 4A MS (3.1 g) in $\mathrm{CH}_{2} \mathrm{Cl}_{2}(30 \mathrm{~mL})$ at $0{ }^{\circ} \mathrm{C}$ was added $\mathrm{MgBr}_{2} \cdot \mathrm{OEt}_{2}(587 \mathrm{mg}, 2.27 \mathrm{mmol})$, and the reaction mixture was stirred at $0{ }^{\circ} \mathrm{C}$ for $3 \mathrm{~h}$. The reaction was quenched with saturated aqueous $\mathrm{NaHCO}_{3}$ and the mixture was extracted with $\mathrm{CH}_{2} \mathrm{Cl}_{2}$. The extract was washed with water, died, and concentrated in vacuo. Purification by flash chromatography (25\% EtOAc in hexane) gave a 1:1 mixture of bromo ketones $8(568 \mathrm{mg}, 60 \%)$. FABMS $m / z 626\left(\mathrm{MH}^{+}\right)$and $628\left(\mathrm{MH}^{+}+2\right)$.

\section{Compound 9}

To a stirred solution of the bromo ketones $8(589 \mathrm{mg}, 0.939 \mathrm{mmol})$ in $\mathrm{CH}_{2} \mathrm{Cl}_{2}(9.3 \mathrm{~mL})$ at $0{ }^{\circ} \mathrm{C}$ was added DBU (165 $\mu \mathrm{L}, 1.033 \mathrm{mmol}$ ), and the solution was stirred at $0{ }^{\circ} \mathrm{C}$ for $35 \mathrm{~min}$. The reaction was quenched with saturated aqueous $\mathrm{NH}_{4} \mathrm{Cl}$ and the mixture was extracted with EtOAc. The extract was washed with water, dried, and concentrated in vacuo. The ${ }^{1} \mathrm{H}$ NMR analysis of the crude product showed a 94:6 mixture of diastereoisomers. Purification by flash chromatography (15\% EtOAc in hexane) gave ketone 9 (462 mg, 90\%) as a colorless oil. [ $\alpha]^{25}{ }_{\mathrm{D}}-7.59$ (c 1.0, $\left.\mathrm{CHCl}_{3}\right)$; IR $\left(\mathrm{CHCl}_{3}\right)$ 1726, 1612, 1513, 1473, 1249, $1101 \mathrm{~cm}^{-1}$; ${ }^{1} \mathrm{H}$ NMR (400 MHz, $\left.\mathrm{C}_{6} \mathrm{D}_{6}\right) \delta 1.08(9 \mathrm{H}, \mathrm{s}), 1.11(9 \mathrm{H}, \mathrm{s}), 1.51(1 \mathrm{H}, \mathrm{q}$, $J=11.7 \mathrm{~Hz}), 1.73(3 \mathrm{H}, \mathrm{s}), 2.08(1 \mathrm{H}, \mathrm{dd}, J=15.1,11.2 \mathrm{~Hz}), 2.38(1 \mathrm{H}, \mathrm{ddd}, J=11.7,3.9,3.9 \mathrm{~Hz}), 2.52(1 \mathrm{H}, \mathrm{ddd}, J=$ 15.1, 7.3, 7.3 Hz), 2.71-2.90 (3H, m), $3.15(1 \mathrm{H}, \mathrm{ddd}, J=9.8,9.8,4.9 \mathrm{~Hz}), 3.31(3 \mathrm{H}, \mathrm{s}), 3.39(1 \mathrm{H}, \mathrm{dd}, J=7.3,4.4 \mathrm{~Hz})$, $3.74(1 \mathrm{H}, \mathrm{ddd}, J=10.7,9.3,4.4 \mathrm{~Hz}), 3.82(1 \mathrm{H}, \mathrm{t}, J=10.2 \mathrm{~Hz}), 3.87(2 \mathrm{H}, \mathrm{s}), 4.13(1 \mathrm{H}, \mathrm{dd}, J=10.2,4.9 \mathrm{~Hz}), 4.36(2 \mathrm{H}$, s), $5.77(1 \mathrm{H}, \mathrm{brt}, J=6.8 \mathrm{~Hz}), 6.80$ and $7.26\left(\right.$ each $2 \mathrm{H}, \mathrm{d}, J=8.3 \mathrm{~Hz}$ ); ${ }^{13} \mathrm{C}$ NMR $\left(100 \mathrm{MHz}, \mathrm{CDCl}_{3}\right) \delta$ 14.1, 19.9, 22.6, $27.0(3 \mathrm{xC}), 27.4(3 \mathrm{xC}), 27.9,38.3,44.9,55.2,66.7,71.1,72.4,75.3,75.6,76.6,77.1,82.9,113.7$ (2xC), 122.7, 129.4 (2xC), 130.6, 134.9, 159.1, 204.8; FABMS $m / z$ $547\left(\mathrm{MH}^{+}\right)$; HRFABMS $m / z$ calcd for $\mathrm{C}_{30} \mathrm{H}_{47} \mathrm{O}_{7} \mathrm{Si}\left(\mathrm{MH}^{+}\right) 547.3088$, 
found 547.3056.

\section{Compound 10}

To a stirred solution of ketone $9(649 \mathrm{mg}, 1.188 \mathrm{mmol})$ in $\mathrm{CH}_{2} \mathrm{Cl}_{2}(12 \mathrm{~mL})$ at $-50{ }^{\circ} \mathrm{C}$ was added $\mathrm{Me}_{3} \mathrm{Al}(4.15 \mathrm{~mL}$ of a 1.43 $\mathrm{M}$ solution in hexane, $5.94 \mathrm{mmol}$ ), and the solution was stirred at $-50{ }^{\circ} \mathrm{C}$ for $3 \mathrm{~h}$ and then at $-20{ }^{\circ} \mathrm{C}$ for $2 \mathrm{~h}$. The reaction was quenched by the careful addition of $\mathrm{MeOH}$ until gas evolution ceased. The mixture was warmed at room temperature, diluted with EtOAc, and washed saturated aqueous sodium potassium tartrate. The extract was washed with water and brine, dried, and concentrated in vacuo. The ${ }^{1} \mathrm{H}$ NMR analysis of the crude product showed a 94:6 mixture of diastereoisomers. Purification by flash chromatography (25\% EtOAc in hexane) gave alcohol 10 (644 mg, $90 \%)$ as a colorless oil. $[\alpha]_{\mathrm{D}}^{25}-34.4\left(c 0.9, \mathrm{CHCl}_{3}\right)$; IR $\left(\mathrm{CHCl}_{3}\right) 3604,3473,1612,1513,1473,1249,1090 \mathrm{~cm}^{-1} ;{ }^{1} \mathrm{H}$ NMR (400 MHz, C $\left.6 \mathrm{D}_{6}\right) \delta 1.09(9 \mathrm{H}, \mathrm{s}), 1.10(9 \mathrm{H}, \mathrm{s}), 1.11(3 \mathrm{H}, \mathrm{s}), 1.46(1 \mathrm{H}, \mathrm{t}, J=11.7 \mathrm{~Hz}), 1.58(1 \mathrm{H}, \mathrm{q}, J=11.7 \mathrm{~Hz})$, $1.72(1 \mathrm{H}, \mathrm{s}, \mathrm{OH}), 1.76(3 \mathrm{H}, \mathrm{s}), 2.01(1 \mathrm{H}, \mathrm{dd}, J=11.7,4.4 \mathrm{~Hz}), 2.15(1 \mathrm{H}, \mathrm{ddd}, J=14.6,8.8,6.4 \mathrm{~Hz}), 2.41(1 \mathrm{H}, \mathrm{ddd}, J=$ 11.2, 8.3, $4.4 \mathrm{~Hz}), 2.52(1 \mathrm{H}, \mathrm{ddd}, J=14.6,7.8,1.5 \mathrm{~Hz}), 2.78(1 \mathrm{H}, \mathrm{ddd}, J=11.2,9.3,3.9 \mathrm{~Hz}), 2.94(1 \mathrm{H}, \mathrm{ddd}, J=11.7$, 9.3, 4.4 Hz), $3.08(1 \mathrm{H}, \mathrm{dd}, J=9.8,2.4 \mathrm{~Hz}), 3.29(1 \mathrm{H}, \mathrm{m}), 3.30(3 \mathrm{H}, \mathrm{s}), 3.77(1 \mathrm{H}, \mathrm{ddd}, J=10.7,8.8,4.4 \mathrm{~Hz}), 3.90(1 \mathrm{H}, \mathrm{t}$, $J=10.1 \mathrm{~Hz}), 3.91(2 \mathrm{H}, \mathrm{s}), 4.25(1 \mathrm{H}, \mathrm{dd}, J=10.1,4.9 \mathrm{~Hz}), 4.38(2 \mathrm{H}, \mathrm{s}), 5.82(1 \mathrm{H}, \mathrm{br} \mathrm{t}, J=6.6 \mathrm{~Hz}), 6.80$ and 7.27 (each $2 \mathrm{H}, \mathrm{d}, J=8.3 \mathrm{~Hz}) ;{ }^{13} \mathrm{C} \mathrm{NMR}\left(100 \mathrm{MHz}, \mathrm{CDCl}_{3}\right) \delta 14.2,19.9,21.8,22.6,27.0(3 \mathrm{xC}), 27.3$ (3xC), 27.4, 38.4, 45.2, 55.2, $66.8,71.1,71.3,72.6,75.7,76.5,77.2,77.6,84.7,113.7$ (2xC), 124.8, 129.3 (2xC), 130.6, 134.0, 159.1; FABMS m/z $563\left(\mathrm{MH}^{+}\right)$; HRFABMS $\mathrm{m} / z$ calcd for $\mathrm{C}_{31} \mathrm{H}_{51} \mathrm{O}_{7} \mathrm{Si}\left(\mathrm{MH}^{+}\right)$563.3401, found 563.3409.

\section{Compound 11}

(i) Removal of the PMB group. To a stirred solution of $\mathbf{1 0}(618 \mathrm{mg}, 1.10 \mathrm{mmol})$ in $\mathrm{CH}_{2} \mathrm{Cl}_{2}(7.3 \mathrm{~mL})$ and $\mathrm{H}_{2} \mathrm{O}(0.36 \mathrm{~mL})$ at $0{ }^{\circ} \mathrm{C}$ was added DDQ (500 mg, $2.20 \mathrm{mmol}$ ), and the reaction mixture was stirred at room temperature for $1.5 \mathrm{~h}$. The reaction mixture was diluted with $\mathrm{MeOH}(7.3 \mathrm{~mL})$ and treated with $\mathrm{CeCl}_{3} \cdot 7 \mathrm{H}_{2} \mathrm{O}(615 \mathrm{mg}, 1.651 \mathrm{mmol})$ and $\mathrm{NaBH}_{4}$ $(624 \mathrm{mg}, 16.42 \mathrm{mmol})$. After stirring at $0^{\circ} \mathrm{C}$ for $1.5 \mathrm{~h}$, the reaction mixture was extracted with EtOAc. The extract was washed with waster and brine, dried, and concentrated in vacuo. Purification by flash chromatography $(60 \%$ EtOAc in hexane) gave allylic alcohol $(447 \mathrm{mg}, 92 \%)$ as a colorless oil. $[\alpha]^{25}-39.5\left(c 0.73, \mathrm{CHCl}_{3}\right) ; \mathrm{IR}\left(\mathrm{CHCl}_{3}\right) 3607,3462$, 1473, 1249, 1091, 1042, $827 \mathrm{~cm}^{-1}$; ${ }^{1} \mathrm{H}$ NMR (600 MHz, $\left.\mathrm{CDCl}_{3}\right) \delta 0.99(9 \mathrm{H}, \mathrm{s}), 1.03(9 \mathrm{H}, \mathrm{s}), 1.26(3 \mathrm{H}, \mathrm{s}), 1.521(1 \mathrm{H}, \mathrm{q}$, $J=11.3 \mathrm{~Hz}), 1.522(1 \mathrm{H}, \mathrm{t}, J=11.7 \mathrm{~Hz}), 1.65(2 \mathrm{H}, \mathrm{br}, 2 \mathrm{xOH}), 1.69(3 \mathrm{H}, \mathrm{s}), 2.07(1 \mathrm{H}, \mathrm{m}), 2.10(1 \mathrm{H}, \mathrm{dd}, J=11.3,4.0$ $\mathrm{Hz}), 2.42-2.48(2 \mathrm{H}, \mathrm{m}), 3.03(1 \mathrm{H}, \mathrm{ddd}, J=11.3,9.2,4.8 \mathrm{~Hz}), 3.10(1 \mathrm{H}, \mathrm{ddd}, J=12.1,9.5,4.8 \mathrm{~Hz}), 3.16(1 \mathrm{H}, \mathrm{dd}, J=$ 9.2, 3.3 Hz), $3.33(1 \mathrm{H}, \mathrm{ddd}, J=10.3,10.3,5.1 \mathrm{~Hz}), 3.81(1 \mathrm{H}, \mathrm{t}, J=10.3 \mathrm{~Hz}), 3.82(1 \mathrm{H}, \mathrm{m}), 4.02(2 \mathrm{H}, \mathrm{s}), 4.13(1 \mathrm{H}, \mathrm{dd}, J$ $=10.3,4.8 \mathrm{~Hz}), 5.55(1 \mathrm{H}$, br t,$J=6.6 \mathrm{~Hz}):{ }^{13} \mathrm{C} \mathrm{NMR}\left(150 \mathrm{MHz}, \mathrm{CDCl}_{3}\right) \delta 13.9,19.9,21.7,22.6,27.0(3 \times \mathrm{C}), 27.2,27.4$ (3xC), 38.4, 45.3, 66.8, 68.7, 71.3, 72.7, 76.5, 77.2, 77.6, 84.7, 122.7, 136.5; FABMS $m / z 443\left(\mathrm{MH}^{+}\right)$; HRFAMS $m / z$ calcd for $\mathrm{C}_{23} \mathrm{H}_{43} \mathrm{O}_{6} \mathrm{Si}\left(\mathrm{MH}^{+}\right)$443.2283, found 443.2297 .

(ii) Epoxidation. To a solution of the allylic alcohol (447 mg, $1.011 \mathrm{mmol})$ in $\mathrm{CH}_{2} \mathrm{Cl}_{2}(10 \mathrm{~mL})$ were added $\mathrm{NaHCO}_{3}$ $(255 \mathrm{mg}, 3.034 \mathrm{mmol})$ and $\mathrm{m}$-CPBA $(262 \mathrm{mg}, 1.517 \mathrm{mmol})$, and the reaction mixture was stirred at $0{ }^{\circ} \mathrm{C}$ for $30 \mathrm{~min}$. The mixture was quenched with saturated aqueous $\mathrm{Na}_{2} \mathrm{~S}_{2} \mathrm{O}_{3}$ and extracted with EtOAc. The extract was washed with saturated aqueous $\mathrm{NaHCO}_{3}$, water, and brine, dried, and concentrated in vacuo. The ${ }^{1} \mathrm{H}$ NMR analysis of the crude product showed a 96:4 mixture of diastereoisomers. Purification by flash chromatography (75\% EtOAc in hexane) gave dihydroxy epoxide 11 (422 mg, 91\%) as a colorless oil. [ $\alpha]_{\mathrm{D}}^{25}-32.7$ (c 1.0, $\left.\mathrm{CHCl}_{3}\right)$; IR $\left(\mathrm{CHCl}_{3}\right) 3599,3451,1473,1387$, 1091, 1042, $827 \mathrm{~cm}^{-1} ;{ }^{1} \mathrm{H}$ NMR (400 MHz, $\left.\mathrm{CDCl}_{3}\right) \delta 0.99(9 \mathrm{H}, \mathrm{s}), 1.03(9 \mathrm{H}, \mathrm{s}), 1.25(3 \mathrm{H}, \mathrm{s}), 1.31(3 \mathrm{H}, \mathrm{s}), 1.541(1 \mathrm{H}, \mathrm{t}$, 
$J=11.2 \mathrm{~Hz}), 1.543(1 \mathrm{H}, \mathrm{q}, J=11.2 \mathrm{~Hz}), 1.74-1.78(1 \mathrm{H}, \mathrm{m}), 1.79(1 \mathrm{H}, \mathrm{br}, \mathrm{OH}), 1.90(1 \mathrm{H}, \mathrm{ddd}, J=14.6,6.8,3.4 \mathrm{~Hz})$, $2.00(1 \mathrm{H}, \mathrm{dd}, J=7.8,3.9 \mathrm{~Hz}, \mathrm{OH}), 2.13(1 \mathrm{H}, \mathrm{dd}, J=11.7,3.9 \mathrm{~Hz}), 2.47(1 \mathrm{H}, \mathrm{ddd}, J=11.3,3.9,3.9 \mathrm{~Hz}), 3.04-3.15(2 \mathrm{H}$, $\mathrm{m}), 3.25(1 \mathrm{H}, \mathrm{t}, J=6.8 \mathrm{~Hz}), 3.26(1 \mathrm{H}, \mathrm{dd}, J=8.7,3.4 \mathrm{~Hz}), 3.33(1 \mathrm{H}, \mathrm{ddd}, J=9.8,9.8,4.9 \mathrm{~Hz}), 3.59(1 \mathrm{H}, \mathrm{dd}, J=12.2$,

$7.8 \mathrm{~Hz}), 3.67(1 \mathrm{H}, \mathrm{dd}, J=12.2,3.9 \mathrm{~Hz}), 3.80(1 \mathrm{H}, \mathrm{t}, J=10.2 \mathrm{~Hz}), 3.83(1 \mathrm{H}, \mathrm{m}), 4.13(1 \mathrm{H}, \mathrm{dd}, J=10.2,4.9 \mathrm{~Hz}):{ }^{13} \mathrm{C}$ NMR (100 MHz, $\left.\mathrm{CDCl}_{3}\right) \delta$ 14.2, 19.9, 21.6, 22.6, 27.0 (3xC), 27.4 (3xC), 28.1, 38.3, 45.4, 58.3, 60.7, 65.5, 66.8, 71.0, 72.7, 76.3, 77.2, 77.6, 82.9; FABMS $m / z 459\left(\mathrm{MH}^{+}\right)$; HRFABMS $m / z$ calcd for $\mathrm{C}_{23} \mathrm{H}_{43} \mathrm{O}_{7} \mathrm{Si} 459,2776$, found 459.2752 .

\section{Compound 12}

(i) Oxidation. To a stirred solution of dihydroxy epoxide 11 (494 mg, $1.079 \mathrm{mmol})$ in $\mathrm{CH}_{2} \mathrm{Cl}_{2}$ (11 mL) and DMSO (2.2 $\mathrm{mL})$ at $0{ }^{\circ} \mathrm{C}$ were added $\mathrm{Et}_{3} \mathrm{~N}(0.76 \mathrm{~mL}, 5.394 \mathrm{mmol})$ and $\mathrm{SO}_{3} \cdot$ pyridine $(515 \mathrm{mg}, 3.236 \mathrm{mmol})$, and the reaction mixture was stirred at $0{ }^{\circ} \mathrm{C}$ for $10 \mathrm{~min}$ and then at room temperature for $1 \mathrm{~h}$. The reaction was quenched with saturated aqueous $\mathrm{NH}_{4} \mathrm{Cl}$ and the mixture was extracted with $\mathrm{Et}_{2} \mathrm{O}$. The extract was washed with water and brine, dried, and concentrated in vacuo to give crude aldehyde (492 $\mathrm{mg}$ ), which was used in the next reaction without further purification.

(ii) Wittig reaction. To a suspension of $\mathrm{Ph}_{3} \mathrm{P}^{+} \mathrm{CH}_{3} \mathrm{Br}^{-}(1.73 \mathrm{~g}, 4.855 \mathrm{mmol})$ in THF $(10 \mathrm{~mL})$ at $0{ }^{\circ} \mathrm{C}$ was added $\mathrm{KHMDS}$ $(6.92 \mathrm{~mL}$ of a $0.67 \mathrm{M}$ solution in toluene, $4.636 \mathrm{mmol})$. The resulting yellow solution was stirred at $0{ }^{\circ} \mathrm{C}$ for $45 \mathrm{~min}$. A solution of the aldehyde $(492 \mathrm{mg})$ in THF $(8 \mathrm{~mL})$ was added to the ylide solution at $-78^{\circ} \mathrm{C}$, and the reaction mixture was stirred for $30 \mathrm{~min}$ and then at room temperature for $1 \mathrm{~h}$. The reaction was quenched with saturated aqueous $\mathrm{NH}_{4} \mathrm{Cl}$ and the mixture was extracted with EtOAc. The extract was washed with water and brine, dried, and concentrated in vacuo. Purification by flash chromatography (30\% EtOAc in hexane) gave hydroxy epoxide 12 (368 mg, $75 \%$ for the two steps) as a colorless oil. $[\alpha]^{25}-59.8\left(c 0.75, \mathrm{CHCl}_{3}\right)$; IR $\left(\mathrm{CHCl}_{3}\right) 3604,3466,1473,1387,1090,1041,827 \mathrm{~cm}^{-1} ;{ }^{1} \mathrm{H}$ NMR (400 MHz, $\left.\mathrm{CDCl}_{3}\right) \delta 0.99(9 \mathrm{H}, \mathrm{s}), 1.03(9 \mathrm{H}, \mathrm{s}), 1.25(3 \mathrm{H}, \mathrm{s}), 1.41(3 \mathrm{H}, \mathrm{s}), 1.48(1 \mathrm{H}, \mathrm{br} \mathrm{s}, \mathrm{OH}), 1.54(1 \mathrm{H}, \mathrm{t}, J=$ $11.2 \mathrm{~Hz}), 1.55(1 \mathrm{H}, \mathrm{q}, J=11.2 \mathrm{~Hz}), 1.76(1 \mathrm{H}, \mathrm{ddd}, J=14.6,9.3,5.4 \mathrm{~Hz}), 1.90(1 \mathrm{H}, \mathrm{ddd}, J=14.6,6.8,2.9 \mathrm{~Hz}), 2.13$ $(1 \mathrm{H}, \mathrm{dd}, J=11.7,4.4 \mathrm{~Hz}), 2.47(1 \mathrm{H}, \mathrm{ddd}, J=11.7,4.4,3.9 \mathrm{~Hz}), 3.05-3.15(3 \mathrm{H}, \mathrm{m}), 3.27(1 \mathrm{H}, \mathrm{dd}, J=9.3,2.9 \mathrm{~Hz}), 3.33$ $(1 \mathrm{H}, \mathrm{ddd}, J=10.3,9.3,4.9 \mathrm{~Hz}), 3.80(1 \mathrm{H}, \mathrm{t}, J=10.2 \mathrm{~Hz}), 3.82(1 \mathrm{H}, \mathrm{m}), 4.13(1 \mathrm{H}, \mathrm{dd}, J=9.8,4.9 \mathrm{~Hz}), 5.19(1 \mathrm{H}, \mathrm{dd}, J$ $=10.7,1.0 \mathrm{~Hz}), 5.33(1 \mathrm{H}, \mathrm{dd}, J=17.6,1.0 \mathrm{~Hz}), 5.67(1 \mathrm{H}, \mathrm{dd}, J=17.6,10.7 \mathrm{~Hz}):{ }^{13} \mathrm{C} \mathrm{NMR}\left(100 \mathrm{MHz}, \mathrm{CDCl}_{3}\right) \delta 14.9$, 19.9, 21.7, 22.6, 27.0 (3xC), 27.4 (3xC), 28.5, 38.3, 45.4, 59.2, 63.1, 66.8, 71.0, 72.7, 76.3, 77.3, 77.6, 82.9, 116.0, 140.8; FABMS $m / z 455\left(\mathrm{MH}^{+}\right)$; HRFABMS $m / z$ calcd for $\mathrm{C}_{24} \mathrm{H}_{43} \mathrm{O}_{6} \mathrm{Si}\left(\mathrm{MH}^{+}\right)$455,2829, found 455.2829.

\section{Compound 13}

To a stirred solution of hydroxy epoxide 11 (368 $\mathrm{mg}, 0.812 \mathrm{mmol})$ in $\mathrm{CH}_{2} \mathrm{Cl}_{2}(8.1 \mathrm{~mL})$ at $0{ }^{\circ} \mathrm{C}$ was added PPTS (106 $\mathrm{mg}, 0.081 \mathrm{mmol})$, and the solution was stirred at $0{ }^{\circ} \mathrm{C}$ for $5 \mathrm{~h}$. The reaction was quenched with $\mathrm{Et}_{3} \mathrm{~N}(0.2 \mathrm{~mL})$ and the mixture was concentrated in vacuo. Purification by flash chromatography (20\% EtOAc in hexane) gave hydroxy olefin $13(272 \mathrm{mg}, 74 \%)$ as a solid. Mp 222-224 ${ }^{\circ} \mathrm{C} ;[\alpha]_{\mathrm{D}}^{25}-20.8\left(c 1.0, \mathrm{CHCl}_{3}\right)$; IR $\left(\mathrm{CHCl}_{3}\right)$ 3607, 3478, 1473, 1385, 1091, 1039, $826 \mathrm{~cm}^{-1} ;{ }^{1} \mathrm{H}$ NMR (400 MHz, $\left.\mathrm{CDCl}_{3}\right) \delta 0.99(9 \mathrm{H}, \mathrm{s}), 1.04(9 \mathrm{H}, \mathrm{s}), 1.36(3 \mathrm{H}, \mathrm{s}), 1.38(3 \mathrm{H}, \mathrm{s}), 1.44(1 \mathrm{H}, \mathrm{t}, J=11.7$ $\mathrm{Hz}), 1.56(1 \mathrm{H}, \mathrm{q}, J=11.7 \mathrm{~Hz}), 1.67(1 \mathrm{H}, \mathrm{d}, J=4.9 \mathrm{~Hz}, \mathrm{OH}), 1.84(1 \mathrm{H}, \mathrm{q}, J=11.7 \mathrm{~Hz}), 2.09(1 \mathrm{H}, \mathrm{ddd}, J=11.7,4.9,3.9$ $\mathrm{Hz}), 2.17(1 \mathrm{H}, \mathrm{dd}, J=11.7,4.8 \mathrm{~Hz}), 2.46(1 \mathrm{H}, \mathrm{ddd}, J=11.7,4.4,3.9 \mathrm{~Hz}), 3.13(1 \mathrm{H}, \mathrm{ddd}, J=11.7,9.3,3.9 \mathrm{~Hz}), 3.19$ $(1 \mathrm{H}, \mathrm{dd}, J=12.2,3.4 \mathrm{~Hz}), 3.27$ (1H, ddd, $J=11.7,9.3,4.8 \mathrm{~Hz}), 3.34$ (1H, ddd, $J=10.2,9.3,4.9 \mathrm{~Hz}), 3.65$ (1H, ddd, $J$ $=11.7,4.9,4.9 \mathrm{~Hz}), 3.80(1 \mathrm{H}, \mathrm{t}, J=10.2 \mathrm{~Hz}), 3.84(1 \mathrm{H}, \mathrm{m}), 4.14(1 \mathrm{H}, \mathrm{dd}, J=9.8,4.9 \mathrm{~Hz}), 5.11(1 \mathrm{H}, \mathrm{dd}, J=10.7,1.0$ $\mathrm{Hz}), 5.28(1 \mathrm{H}, \mathrm{dd}, J=17.1,1.0 \mathrm{~Hz}), 5.90(1 \mathrm{H}, \mathrm{dd}, J=17.1,10.7 \mathrm{~Hz}):{ }^{13} \mathrm{C} \mathrm{NMR}\left(100 \mathrm{MHz}, \mathrm{CDCl}_{3}\right) \delta 19.9,20.5,20.6$, 22.6, $27.1(3 \times C), 27.4(3 \times C), 30.2,38.5,43.7,66.8,72.9,73.3,73.9,77.4,77.7,78.0,78.7,80.8,113.3$, 144.6; FABMS 
$m / z 455\left(\mathrm{MH}^{+}\right)$; HRFABMS $m / z$ calcd for $\mathrm{C}_{24} \mathrm{H}_{43} \mathrm{O}_{6} \mathrm{Si}\left(\mathrm{MH}^{+}\right) 455,2829$, found 455.2838. Anal. Calcd for $\mathrm{C}_{24} \mathrm{H}_{42} \mathrm{O}_{6} \mathrm{Si}$ : C, $63.40 ; \mathrm{H}, 9.32$, found $\mathrm{C}, 63.67 ; \mathrm{H}, 9.49$.

\section{Compound 14}

To a solution of hydroxy olefin $13(362 \mathrm{mg}, 0.797 \mathrm{mmol})$ in $\mathrm{CH}_{2} \mathrm{Cl}_{2}(16 \mathrm{~mL})$ were added $\mathrm{VO}(\mathrm{acac})_{2}(4.2 \mathrm{mg}, 0.016$ $\mathrm{mmol})$ and $t$ - $\mathrm{BuOOH}\left(0.50 \mathrm{~mL}\right.$ of a $7.99 \mathrm{M}$ solution in $\left.(t-\mathrm{Bu})_{2} \mathrm{O}, 3.986 \mathrm{mmol}\right)$, and the solution was heated at $35{ }^{\circ} \mathrm{C}$ for $24 \mathrm{~h}$. After cooling to room temperature, the reaction was quenched with saturated aqueous $\mathrm{Na}_{2} \mathrm{~S}_{2} \mathrm{O}_{3}$ and the mixture was extracted with $\mathrm{CH}_{2} \mathrm{Cl}_{2}$. The extract was washed with saturated aqueous $\mathrm{NaHCO}_{3}$ and water, dried, and concentrated in vacuo. Purification by flash chromatography (8\% acetone in $\left.\mathrm{CH}_{2} \mathrm{Cl}_{2}\right)$ gave hydroxy epoxide $\mathbf{1 4}(319 \mathrm{mg}, 85 \%)$ as a colorless oil. $[\alpha]^{25}{ }_{\mathrm{D}}-25.8\left(\mathrm{c} 0.77, \mathrm{CHCl}_{3}\right)$; IR $\left(\mathrm{CHCl}_{3}\right) 3607,1473,1385,1105,1090,827 \mathrm{~cm}^{-1}$; ${ }^{1} \mathrm{H}$ NMR $(400 \mathrm{MHz}$, $\left.\mathrm{CDCl}_{3}\right) \delta 0.99(9 \mathrm{H}, \mathrm{s}), 1.04(9 \mathrm{H}, \mathrm{s}), 1.20(3 \mathrm{H}, \mathrm{s}), 1.32(3 \mathrm{H}, \mathrm{s}), 1.39(1 \mathrm{H}, \mathrm{t}, J=11.2 \mathrm{~Hz}), 1.55(1 \mathrm{H}, \mathrm{q}, J=11.2 \mathrm{~Hz}), 1.82$ $(1 \mathrm{H}, \mathrm{q}, J=12.2 \mathrm{~Hz}), 2.03(1 \mathrm{H}, \mathrm{br}, \mathrm{OH}), 2.09(1 \mathrm{H}, \mathrm{m}), 2.13(1 \mathrm{H}, \mathrm{dd}, J=11.2,4.4 \mathrm{~Hz}), 2.46(1 \mathrm{H}, \mathrm{ddd}, J=11.2,4.4,3.9$ Hz), $2.68(1 \mathrm{H}, \mathrm{t}, J=4.4 \mathrm{~Hz}), 2.77(1 \mathrm{H}, \mathrm{dd}, J=4.4,2.4 \mathrm{~Hz}), 3.00(1 \mathrm{H}, \mathrm{dd}, J=3.9,2.4 \mathrm{~Hz}), 3.12(1 \mathrm{H}, \mathrm{m}), 3.14(1 \mathrm{H}, \mathrm{dd}, J$ $=12.2,3.9 \mathrm{~Hz}), 3.25(1 \mathrm{H}, \mathrm{ddd}, J=11.2,9.3,4.4 \mathrm{~Hz}), 3.33(1 \mathrm{H}, \mathrm{ddd}, J=10.2,9.3,4.9 \mathrm{~Hz}), 3.80(1 \mathrm{H}, \mathrm{t}, J=10.2 \mathrm{~Hz})$, $3.83(1 \mathrm{H}, \mathrm{m}), 3.91(1 \mathrm{H}, \mathrm{dd}, J=12.2,4.9 \mathrm{~Hz}), 4.14(1 \mathrm{H}, \mathrm{dd}, \mathrm{J}=10.2,4.9 \mathrm{~Hz}):{ }^{13} \mathrm{C} \mathrm{NMR}\left(100 \mathrm{MHz}, \mathrm{CDCl}_{3}\right) \delta 17.6,19.9$, 20.2, 22.6, $27.1(3 \mathrm{xC}), 27.4(3 \mathrm{xC}), 29.8,38.4,43.4,58.8,66.8,72.8,72.9,73.5,75.3,76.1,77.2,78.1,78.6,80.4$; FABMS $m / z 471\left(\mathrm{MH}^{+}\right)$; HRFABMS $m / z$ calcd for $\mathrm{C}_{24} \mathrm{H}_{43} \mathrm{O}_{7} \mathrm{Si}\left(\mathrm{MH}^{+}\right)$471.2778, found 471.2808.

\section{Compound 15}

To a solution of hydroxy epoxide $13(108 \mathrm{mg}, 1.944 \mathrm{mmol})$ in $\mathrm{CH}_{2} \mathrm{Cl}_{2}(15 \mathrm{~mL})$ and $\mathrm{Et}_{3} \mathrm{~N}(0.24 \mathrm{~mL}, 1.727 \mathrm{mmol})$ were added DMAP $(3.0 \mathrm{mg})$ and TES-Cl $(174 \mu \mathrm{L}, 1.039 \mathrm{mmol})$, and the solution was stirred at room temperature for $18 \mathrm{~h}$. The reaction was quenched with $\mathrm{MeOH}(0.2 \mathrm{~mL})$ and the mixture was extracted with EtOAc. The extract was washed with water, saturated aqueous $\mathrm{NaHCO}_{3}$, and brine, dried, and concentrated in vacuo. Purification by flash chromatography (8\% EtOAc in hexane) gave epoxide $15(130 \mathrm{mg}, 96 \%)$ as a colorless oil. $[\alpha]_{\mathrm{D}}^{25}-28.2\left(c 0.84, \mathrm{CHCl}_{3}\right)$; IR $\left(\mathrm{CHCl}_{3}\right)$ 1473, 1092, 1071, $827 \mathrm{~cm}^{-1} ;{ }^{1} \mathrm{H} \mathrm{NMR}\left(400 \mathrm{MHz}, \mathrm{CDCl}_{3}\right) \delta 0.63(6 \mathrm{H}, \mathrm{q}, J=7.8 \mathrm{~Hz}), 0.97(9 \mathrm{H}, \mathrm{t}, J=7.8 \mathrm{~Hz})$, $0.99(9 \mathrm{H}, \mathrm{s}), 1.04(9 \mathrm{H}, \mathrm{s}), 1.29(1 \mathrm{H}, \mathrm{t}, J=11.7 \mathrm{~Hz}), 1.30(3 \mathrm{H}, \mathrm{s}), 1.36(3 \mathrm{H}, \mathrm{s}), 1.54(1 \mathrm{H}, \mathrm{q}, J=11.7 \mathrm{~Hz}), 1.85(1 \mathrm{H}, \mathrm{q}, J$ $=11.7 \mathrm{~Hz}), 1.99(1 \mathrm{H}, \mathrm{ddd}, J=11.7,4.9,3.9 \mathrm{~Hz}), 2.14(1 \mathrm{H}, \mathrm{dd}, J=11.7,4.9 \mathrm{~Hz}), 2.45(1 \mathrm{H}$, ddd, $J=11.7,4.4,3.9 \mathrm{~Hz})$, $2.61(1 \mathrm{H}, \mathrm{dd}, J=5.9,3.9 \mathrm{~Hz}), 2.81(1 \mathrm{H}, \mathrm{dd}, J=5.9,2.4 \mathrm{~Hz}), 3.01-3.06(2 \mathrm{H}, \mathrm{m}), 3.09(1 \mathrm{H}, \mathrm{ddd}, J=11.7,9.3,3.9 \mathrm{~Hz})$, $3.23(1 \mathrm{H}$, ddd, $J=11.7,9.3,4.9 \mathrm{~Hz}), 3.32(1 \mathrm{H}, \mathrm{ddd}, J=10.3,9.8,4.9 \mathrm{~Hz}), 3.77(1 \mathrm{H}, \mathrm{dd}, J=11.2,4.9 \mathrm{~Hz}), 3.79(1 \mathrm{H}, \mathrm{t}$, $J=10.3 \mathrm{~Hz}), 3.82(1 \mathrm{H}, \mathrm{m}), 4.14(1 \mathrm{H}, \mathrm{dd}, J=10.3,4.9 \mathrm{~Hz}):{ }^{13} \mathrm{C}$ NMR (100 MHz, $\left.\mathrm{CDCl}_{3}\right) \delta 5.1(3 \mathrm{xC}), 6.9(3 \mathrm{xC}), 19.9$, 20.1, 22.6, $27.1(3 \mathrm{xC}), 27.4(3 \mathrm{xC}), 31.2,38.5,43.4,43.6,59.2,66.8,72.5,72.9,74.9,77.2,77.3,78.0,78.6(2 \times C), 80.3$; FABMS $m / z 585\left(\mathrm{MH}^{+}\right)$; HRFABMS $m / z$ calcd for $\mathrm{C}_{30} \mathrm{H}_{57} \mathrm{O}_{7} \mathrm{Si}_{2}\left(\mathrm{MH}^{+}\right)$585.3643, found 585.3630.

\section{Compound 18}

To a stirred solution of dithiane $\mathbf{1 6 b}(718 \mathrm{mg}, 1.709 \mathrm{mmol})$ in THF at $0{ }^{\circ} \mathrm{C}$ was added $n$-BuLi $(1.29 \mathrm{~mL}$ of a $1.59 \mathrm{M}$ solution in hexane, $2.051 \mathrm{mmol}$ ). After stirring for $10 \mathrm{~min}$, a solution of epoxide 15 (200 mg, $0.342 \mathrm{mmol}$ ) in THF (4 $\mathrm{mL}$ ) was added, and the reaction mixture was stirred at $0{ }^{\circ} \mathrm{C}$ for $35 \mathrm{~min}$. The reaction was quenched with saturated aqueous $\mathrm{NH}_{4} \mathrm{Cl}$ and the mixture was extracted with EtOAc. The extract was washed with water and brine, dried, and concentrated in vacuo. Purification by flash chromatography (6 to15\% EtOAc in hexane) gave hydroxy dithiane 18 (312 $\mathrm{mg}, 91 \%)$ as a colorless oil. $[\alpha]_{\mathrm{D}}^{25}-26.5\left(c 1.0, \mathrm{CHCl}_{3}\right) ; \mathrm{IR}\left(\mathrm{CHCl}_{3}\right) 3503,1473,1092,1071,826 \mathrm{~cm}^{-1} ;{ }^{1} \mathrm{H}$ NMR $(400$ 
$\left.\mathrm{MHz} \mathrm{CDCl}_{3}\right) \delta 0.64(6 \mathrm{H}, \mathrm{q}, J=7.8 \mathrm{~Hz}), 0.96(9 \mathrm{H}, \mathrm{t}, J=7.8 \mathrm{~Hz}), 0.98(9 \mathrm{H}, \mathrm{s}), 1.04(9 \mathrm{H}, \mathrm{s}), 1.29(3 \mathrm{H}, \mathrm{s}), 1.30(1 \mathrm{H}, \mathrm{m})$, $1.31(3 \mathrm{H}, \mathrm{s}), 1.52(1 \mathrm{H}, \mathrm{q}, J=11.2 \mathrm{~Hz}), 1.78-1.98(6 \mathrm{H}, \mathrm{m}), 2.00-2.10(4 \mathrm{H}, \mathrm{m}), 2.40(1 \mathrm{H}, \mathrm{d}, J=15.1 \mathrm{~Hz}), 2.44(1 \mathrm{H}, \mathrm{ddd}$, $J=11.2,4.4,3.9 \mathrm{~Hz}), 2.69-2.90(4 \mathrm{H}, \mathrm{m}), 3.10-3.09(5 \mathrm{H}, \mathrm{m}), 3.22(1 \mathrm{H}, \mathrm{ddd}, J=11.2,9.3,4.4 \mathrm{~Hz}), 3.31(1 \mathrm{H}, \mathrm{ddd}, J=$ $10.2,9.3,4.9 \mathrm{~Hz}), 3.73(1 \mathrm{H}, \mathrm{d}, J=8.8 \mathrm{~Hz}), 3.77(1 \mathrm{H}, \mathrm{t}, J=10.2 \mathrm{~Hz}), 3.79(1 \mathrm{H}, \mathrm{m}), 4.07(1 \mathrm{H}, \mathrm{dd}, J=11.2,5.4 \mathrm{~Hz}), 4.13$ $(1 \mathrm{H}, \mathrm{dd}, J=10.2,4.9 \mathrm{~Hz}), 7.21-7.30(9 \mathrm{H}, \mathrm{m}), 7.42-7.45(6 \mathrm{H}, \mathrm{m}):{ }^{13} \mathrm{C} \mathrm{NMR}\left(100 \mathrm{MHz}, \mathrm{CDCl}_{3}\right) \delta 5.6(3 \mathrm{xC}), 7.0(3 \times \mathrm{C})$, 18.1, 19.9, 20.2, 22.6, 23.3, 25.1, 26.1, 27.1 (3xC), 27.4 (3xC), 31.4, 35.9, 38.4, 39.2, 41.6, 43.6, 53.3, 63.8, 66.8, 72.8, 73.1, 76.1, 77.1, 77.2, 78.0, 78.4, 79.4, 80.1, 86.3, 126.7 (3xC), 127.7 (6xC), 128.7 (6xC), 144.5 (3xC); FABMS m/z $1005\left(\mathrm{MH}^{+}\right)$; HRFABMS $m / z$ calcd for $\mathrm{C}_{56} \mathrm{H}_{85} \mathrm{O}_{8} \mathrm{~S}_{2} \mathrm{Si}_{2}\left(\mathrm{MH}^{+}\right)$1005.5219, found 1005.5251 .

\section{Compound 19}

(i) Deprotection. A solution of dithiane $18(312 \mathrm{mg}, 0.311 \mathrm{mmol})$ and $p$ - $\mathrm{TsOH} \cdot \mathrm{H}_{2} \mathrm{O}(6.5 \mathrm{mg}, 0.034 \mathrm{mmol})$ in $\mathrm{CH}_{2} \mathrm{Cl}_{2}$ $(1.7 \mathrm{~mL})$ and $\mathrm{MeOH}(1.7 \mathrm{~mL})$ was stirred at room temperature for $70 \mathrm{~min}$. The reaction was quenched with $\mathrm{Et}_{3} \mathrm{~N}(0.1$ $\mathrm{mL})$ and the reaction mixture was concentrated in vacuo. Purification by flash chromatography (60\% EtOAc in hexane) gave trihydroxy dithiane $(201 \mathrm{mg}, 100 \%)$ as a solid. Mp $162-164{ }^{\circ} \mathrm{C} ;[\alpha]^{25}-39.7\left(c 0.62, \mathrm{CHCl}_{3}\right) ; \mathrm{IR}_{\mathrm{D}}\left(\mathrm{CHCl}_{3}\right)$ 3480, 1473, 1090, 1041, $826 \mathrm{~cm}^{-1}$; ${ }^{1} \mathrm{H}$ NMR (400 MHz, $\mathrm{CDCl}_{3}$ ) $\delta 0.99$ (9H, s), 1.03 (9H, s), 1.29 (3H, s), $1.30(3 \mathrm{H}, \mathrm{s})$, $1.31(1 \mathrm{H}, \mathrm{t}, J=11.7 \mathrm{~Hz}), 1.55(1 \mathrm{H}, \mathrm{q}, J=11.7 \mathrm{~Hz}), 1.71-2.10(10 \mathrm{H}, \mathrm{m}), 2.20(1 \mathrm{H}, \mathrm{dd}, J=14.6,9.3 \mathrm{~Hz}), 2.40(1 \mathrm{H}, \mathrm{d}, J$ $=14.6 \mathrm{~Hz}), 2.46(1 \mathrm{H}, \mathrm{ddd}, J=11.7,4.4,3.9 \mathrm{~Hz}), 2.74(2 \mathrm{H}, \mathrm{ddd}, J=14.2,5.9,2.9 \mathrm{~Hz}), 2.94-3.05(2 \mathrm{H}, \mathrm{m}), 3.08-3.15$ $(2 \mathrm{H}, \mathrm{m}), 3.24(1 \mathrm{H}, \mathrm{ddd}, J=11.7,9.3,4.9 \mathrm{~Hz}), 3.33(1 \mathrm{H}, \mathrm{ddd}, J=10.2,9.3,4.9 \mathrm{~Hz}), 3.60-3.72(4 \mathrm{H}, \mathrm{m}), 3.79(1 \mathrm{H}, \mathrm{t}, J$ $=10.2 \mathrm{~Hz}), 3.82(2 \mathrm{H}, \mathrm{m}), 4.13(2 \mathrm{H}, \mathrm{m}):{ }^{13} \mathrm{C} \mathrm{NMR}\left(100 \mathrm{MHz}, \mathrm{CDCl}_{3}\right) \delta$ 16.2, 19.9, 20.3, 22.6, 24.9, 25.9, 26.2, 26.4, $27.0(3 \mathrm{xC}), 27.4(3 \mathrm{xC}), 29.1,35.7,37.4,38.4,43.5,51.7,62.4,66.8,72.8,73.3,75.8,77.2(2 \times C), 78.0,78.5,78.7,80.7$; FABMS $m / z 649\left(\mathrm{MH}^{+}\right)$; HRFABMS $m / z$ calcd for $\mathrm{C}_{31} \mathrm{H}_{57} \mathrm{O}_{8} \mathrm{~S}_{2} \mathrm{Si}\left(\mathrm{MH}^{+}\right)$649.3261, found 649.3286. Anal. Calcd for $\mathrm{C}_{31} \mathrm{H}_{56} 0_{8} \mathrm{~S}_{2} \mathrm{Si}: \mathrm{C}, 57.38 ; \mathrm{H}, 8.71$, found C, 57.03; H, 8.95.

(ii) Pivaloylation. To a stirred solution of the trihydroxy dithiane $(201 \mathrm{mg}, 0.311 \mathrm{mmol})$ in pyridine $(3.1 \mathrm{~mL})$ were added DMAP (1.9 mg, $0.015 \mathrm{mmol})$ and pivaloyl chloride $(0.57 \mathrm{~mL}, 0.466 \mathrm{mmol})$, and the reaction mixture was stirred at $0{ }^{\circ} \mathrm{C}$ for $1.5 \mathrm{~h}$. The reaction was quenched with saturated aqueous $\mathrm{NaHCO}_{3}$ and the mixture was extracted with EtOAc. The extract was washed with water and brine, dried, and concentrated in vacuo. Purification by flash chromatography $\left(35 \%\right.$ EtOAc in hexane) gave pivaloyl dithiane $(227 \mathrm{mg}, 100 \%)$ as a solid. Mp $133-134{ }^{\circ} \mathrm{C}$; $[\alpha]^{25} \mathrm{D}$ -39.7 (c 0.62, $\left.\mathrm{CHCl}_{3}\right)$; IR $\left(\mathrm{CHCl}_{3}\right) 3503,1720,1473,1287,1091,1041,826 \mathrm{~cm}^{-1} ;{ }^{1} \mathrm{H} \mathrm{NMR}\left(400 \mathrm{MHz}, \mathrm{CDCl}_{3}\right) \delta 0.98$ $(9 \mathrm{H}, \mathrm{s}), 1.03(9 \mathrm{H}, \mathrm{s}), 1.20(9 \mathrm{H}, \mathrm{s}), 1.26(1 \mathrm{H}, \mathrm{m}), 1.30(3 \mathrm{H}, \mathrm{s}), 1.31(3 \mathrm{H}, \mathrm{s}), 1.54(1 \mathrm{H}, \mathrm{q}, J=11.7 \mathrm{~Hz}), 1.78(1 \mathrm{H}, \mathrm{q}, J=$ $12.2 \mathrm{~Hz}), 1.82-2.13(8 \mathrm{H}, \mathrm{m}), 2.24-2.36(2 \mathrm{H}, \mathrm{m}), 2.45(1 \mathrm{H}, \mathrm{ddd}, J=11.7,4.4,3.9 \mathrm{~Hz}), 2.71-2.80(2 \mathrm{H}, \mathrm{m}), 2.95-3.08$ $(3 \mathrm{H}, \mathrm{m}), 3.10(1 \mathrm{H}, \mathrm{dd}, J=12.2,3.4 \mathrm{~Hz}), 3.23(1 \mathrm{H}, \mathrm{ddd}, J=11.2,9.3,4.4 \mathrm{~Hz}), 3.31(1 \mathrm{H}, \mathrm{ddd}, J=10.3,9.3,4.9 \mathrm{~Hz})$, 3.59-3.64 (2H, m), $3.77(1 \mathrm{H}, \mathrm{t}, J=10.3 \mathrm{~Hz}), 3.78-3.84(2 \mathrm{H}, \mathrm{m}), 3.98(1 \mathrm{H}, \mathrm{d}, J=2.0 \mathrm{~Hz}), 4.04(2 \mathrm{H}, \mathrm{t}, J=6.3 \mathrm{~Hz}), 4.10$ $(1 \mathrm{H}, \mathrm{dd}, J=10.3,4.9 \mathrm{~Hz}):{ }^{13} \mathrm{C} \mathrm{NMR}\left(100 \mathrm{MHz}, \mathrm{CDCl}_{3}\right) \delta 16.7,19.9,20.3,22.5,22.6,24.8,25.8,26.6,27.1$ (3xC), 27.3 (3xC), $27.4(3 \mathrm{xC}), 29.0,35.9,37.1,38.5,38.8,43.5,51.3,64.0,66.8,72.9,73.3,75.9,76.9,77.2,78.0,78.6,78.9,80.8$, 178.5; FABMS $m / z 733\left(\mathrm{MH}^{+}\right)$; HRFABMS $m / z$ calcd for $\mathrm{C}_{36} \mathrm{H}_{65} \mathrm{O}_{9} \mathrm{~S}_{2} \mathrm{Si}\left(\mathrm{MH}^{+}\right)$733.3839, found 733.3840. Anal. Calcd for $\mathrm{C}_{36} \mathrm{H}_{64} 0_{9} \mathrm{~S}_{2} \mathrm{Si}$ : C, 58.98; $\mathrm{H}, 8.81$, found $\mathrm{C}, 58.61 ; \mathrm{H}, 8.68$.

(iii) Hydrolysis of the dithioacetal. To a stirred suspension of the pivaloyl dithiane $\left(227 \mathrm{mg}, 0.311 \mathrm{mmol}^{2} \mathrm{and} \mathrm{CaCO}_{3}\right.$ (314 mg, $3.108 \mathrm{mmol})$ in THF $(5 \mathrm{~mL})$ and $\mathrm{H}_{2} \mathrm{O}(1.2 \mathrm{~mL})$ was added $\mathrm{Hg}\left(\mathrm{ClO}_{4}\right)_{2} \cdot 6 \mathrm{H}_{2} \mathrm{O}(316 \mathrm{mg}, 0.622 \mathrm{mmol})$, and the reaction mixture was stirred at $0{ }^{\circ} \mathrm{C}$ for $10 \mathrm{~min}$. The reaction mixture was diluted with EtOAc and filtered through a plug of Celite. The filtrate was washed with water and brine, dried, and concentrated in vacuo. Purification by flash 
chromatography (40\% EtOAc in hexane) gave dihydroxy ketone $19(178 \mathrm{mg}, 89 \%)$ as a solid. Mp $176-179{ }^{\circ} \mathrm{C} ;[\alpha]^{25} \mathrm{D}$ -31.8 (c 0.93, $\left.\mathrm{CHCl}_{3}\right)$; IR $\left(\mathrm{CHCl}_{3}\right) 3513,1721,1473,1166,1090,1043,826 \mathrm{~cm}^{-1} ;{ }^{1} \mathrm{H}$ NMR $\left(400 \mathrm{MHz}, \mathrm{CDCl}_{3}\right) \delta 0.99$ $(9 \mathrm{H}, \mathrm{s}), 1.04(9 \mathrm{H}, \mathrm{s}), 1.20(9 \mathrm{H}, \mathrm{s}), 1.25(3 \mathrm{H}, \mathrm{s}), 1.27(1 \mathrm{H}, \mathrm{m}), 1.28(3 \mathrm{H}, \mathrm{s}), 1.54(1 \mathrm{H}, \mathrm{q}, J=11.2 \mathrm{~Hz}), 1.77(1 \mathrm{H}, \mathrm{q}, J=$ $12.2 \mathrm{~Hz}), 1.92(2 \mathrm{H}$, quint, $J=6.8 \mathrm{~Hz}), 1.98-2.09(2 \mathrm{H}, \mathrm{m}), 2.44(1 \mathrm{H}, \mathrm{dd}, J=18.1,10.3 \mathrm{~Hz}), 2.46(1 \mathrm{H}, \mathrm{m}), 2.54(2 \mathrm{H}$, ddd, $J=7.3,7.3,1.5 \mathrm{~Hz}), 2.91(1 \mathrm{H}, \mathrm{dd}, J=18.1,2.0 \mathrm{~Hz}), 3.08(1 \mathrm{H}, \mathrm{dd}, J=12.2,3.4 \mathrm{~Hz}), 3.10(1 \mathrm{H}, \mathrm{m}), 3.23(1 \mathrm{H}, \mathrm{ddd}, J=$ 11.2, 9.3, $4.9 \mathrm{~Hz}), 3.32(1 \mathrm{H}, \mathrm{ddd}, J=10.2,9.3,4.9 \mathrm{~Hz}), 3.46(1 \mathrm{H}, \mathrm{d}, J=1.5 \mathrm{~Hz}, \mathrm{OH}), 3.68(1 \mathrm{H}, \mathrm{d}, J=2.4 \mathrm{~Hz}, \mathrm{OH})$, $3.79(1 \mathrm{H}, \mathrm{t}, J=10.2 \mathrm{~Hz}), 3.80-3.85(2 \mathrm{H}, \mathrm{m}), 3.87(1 \mathrm{H}, \mathrm{ddd}, J=10.3,2.0,2.0 \mathrm{~Hz}), 4.07(2 \mathrm{H}, \mathrm{t}, J=6.8 \mathrm{~Hz}), 4.12(1 \mathrm{H}$, $\mathrm{dd}, J=10.2,4.9 \mathrm{~Hz}):{ }^{13} \mathrm{C}$ NMR $\left(100 \mathrm{MHz}, \mathrm{CDCl}_{3}\right) \delta 16.6,19.9,20.3,22.5,22.6,27.1(3 \mathrm{xC}), 27.2(3 \mathrm{xC}), 27.4(3 \mathrm{xC})$, 29.1, 38.4, 38.8, 39.8, 42.9, 43.6, 63.3, 66.8, 72.9, 73.3, 75.6, 76.4, 77.2, 77.5, 78.1, 78.6, 80.7, 178.5, 212.1; FABMS $m / z 643\left(\mathrm{MH}^{+}\right)$; HRFABMS $m / z$ calcd for $\mathrm{C}_{33} \mathrm{H}_{59} \mathrm{O}_{10} \mathrm{Si}\left(\mathrm{MH}^{+}\right)$643.3874, found 643.3862. Anal. Calcd for $\mathrm{C}_{36} \mathrm{H}_{58} \mathrm{O}_{10} \mathrm{Si}: \mathrm{C}$, 61.64; H, 9.10, found C, 61.89; H, 9.27.

\section{Compound 20}

To a solution of dihydroxy ketone $19(97 \mathrm{mg}, 0.151 \mathrm{mmol})$ in $\mathrm{CH}_{2} \mathrm{Cl}_{2}(1.5 \mathrm{~mL})$ at $-40{ }^{\circ} \mathrm{C}$ were added $\mathrm{Et}_{3} \mathrm{SiH}(0.120 \mathrm{~mL}$, $0.756 \mathrm{mmol})$ and $\mathrm{SnCl}_{4}\left(0.450 \mathrm{~mL}\right.$ of a $1.0 \mathrm{M}$ solution in $\left.\mathrm{CH}_{2} \mathrm{Cl}_{2}, 0.453 \mathrm{mmol}\right)$, and the reaction mixture was stirred at $-40{ }^{\circ} \mathrm{C}$ for $30 \mathrm{~min}$. The reaction was quenched with saturated aqueous $\mathrm{NaHCO}_{3}$ and the mixture was extracted with EtOAc. The extract was washed with water and brine, dried, and concentrated in vacuo. Purification by flash chromatography (20\% EtOAc in hexane) gave alcohol $20(86 \mathrm{mg}, 91 \%)$ as a solid. Mp $181-182{ }^{\circ} \mathrm{C} ;[\alpha]^{25}{ }_{\mathrm{D}}-5.29(c 1.88$, $\left.\mathrm{CHCl}_{3}\right)$; IR $\left(\mathrm{CHCl}_{3}\right) 3559,1719,1473,1387,1287,1166,1090,1043,826 \mathrm{~cm}^{-1} ;{ }^{1} \mathrm{H}$ NMR $\left(400 \mathrm{MHz}, \mathrm{CDCl}_{3}\right) \delta 0.99$ $(9 \mathrm{H}, \mathrm{s}), 1.04(9 \mathrm{H}, \mathrm{s}), 1.19(9 \mathrm{H}, \mathrm{s}), 1.33(3 \mathrm{H}, \mathrm{s}), 1.37(3 \mathrm{H}, \mathrm{s}), 1.39(1 \mathrm{H}, \mathrm{t}, J=11.2 \mathrm{~Hz}), 1.44-1.52(2 \mathrm{H}, \mathrm{m}), 1.55(1 \mathrm{H}, \mathrm{q}, J$ $=11.2 \mathrm{~Hz}), 1.66(1 \mathrm{H}, \mathrm{m}), 1.72-1.82(3 \mathrm{H}, \mathrm{m}), 1.87(1 \mathrm{H}, \mathrm{q}, J=11.7 \mathrm{~Hz}), 2.04(1 \mathrm{H}, \mathrm{ddd}, J=11.7,3.4,3.4 \mathrm{~Hz}), 2.13(1 \mathrm{H}$, $\mathrm{dd}, J=11.7,4.9 \mathrm{~Hz}), 2.47(1 \mathrm{H}, \mathrm{ddd}, J=11.2,4.4,4.4 \mathrm{~Hz}), 2.60(1 \mathrm{H}, \mathrm{d}, J=1.5 \mathrm{~Hz}, \mathrm{OH}), 3.12(1 \mathrm{H}, \mathrm{ddd}, J=11.2,9.3$, $4.4 \mathrm{~Hz}), 3.17(1 \mathrm{H}, \mathrm{dd}, J=11.2,3.4 \mathrm{~Hz}), 3.29(1 \mathrm{H}, \mathrm{ddd}, J=11.2,9.3,4.4 \mathrm{~Hz}), 3.34(1 \mathrm{H}$, ddd, $J=9.3,9.3,4.9 \mathrm{~Hz}), 3.72$ $(1 \mathrm{H}, \mathrm{t}, J=2.4 \mathrm{~Hz}), 3.77(2 \mathrm{H}, \mathrm{m}), 3.79(1 \mathrm{H}, \mathrm{t}, J=10.3 \mathrm{~Hz}), 3.83(1 \mathrm{H}, \mathrm{ddd}, J=10.7,9.3,4.9 \mathrm{~Hz}), 4.05(2 \mathrm{H}, \mathrm{t}, J=6.3$ $\mathrm{Hz}), 4.14(1 \mathrm{H}, \mathrm{dd}, J=10.3,4.9 \mathrm{~Hz}):{ }^{13} \mathrm{C} \mathrm{NMR}\left(100 \mathrm{MHz}, \mathrm{CDCl}_{3}\right) \delta 19.9,20.3,21.3,22.6,24.9,27.0,27.1(3 \mathrm{xC}), 27.2$ (3xC), 27.4 (3xC), 32.0, 35.4, 38.4, 38.7, 43.4, 64.1, 66.8, 70.7, 72.9, 73.8, 74.4, 75.1, 75.9, 77.3, 78.1, 79.0, 82.8, 178.5; FABMS $m / z 627\left(\mathrm{MH}^{+}\right)$; HRFABMS $m / z$ calcd for $\mathrm{C}_{33} \mathrm{H}_{59} \mathrm{O}_{9} \mathrm{Si}\left(\mathrm{MH}^{+}\right)$627.3928, found 627.3943. Anal. Calcd for $\mathrm{C}_{33} \mathrm{H}_{58} 0_{9} \mathrm{Si}: \mathrm{C}, 63.22 ; \mathrm{H}, 9.33$, found $\mathrm{C}, 63.53 ; \mathrm{H}, 9.21$.

\section{Compound 22}

(i) Removal of the pivaloyl group. To a solution of alcohol $20(95 \mathrm{mg}, 0.152 \mathrm{mmol})$ in $\mathrm{CH}_{2} \mathrm{Cl}_{2}(1.5 \mathrm{~mL})$ at $-78{ }^{\circ} \mathrm{C}$ was added DIBALH $(0.560 \mathrm{~mL}$ of a $0.95 \mathrm{M}$ solution in hexane, $0.533 \mathrm{mmol})$, and the reaction mixture was stirred at $-80{ }^{\circ} \mathrm{C}$ for $30 \mathrm{~min}$. The reaction was quenched carefully with $\mathrm{MeOH}(0.5 \mathrm{~mL})$. The mixture was warmed to room temperature, and diluted with EtOAc. A solution of saturated aqueous sodium potassium tartrate was added and the mixture was extracted with EtOAc. The extract was washed with water and brine, dried, and concentrated in vacuo. Purification by flash chromatography (90\% EtOAc in hexane) gave diol (81 mg, 98\%) as a solid. Mp 229-230 ${ }^{\circ} \mathrm{C} ;[\alpha]^{25} \mathrm{D}-6.82(c 1.54$, $\mathrm{CHCl}_{3}$ ); IR $\left(\mathrm{CHCl}_{3}\right) 3558,3436,1473,1387,1090,1043,826 \mathrm{~cm}^{-1} ;{ }^{1} \mathrm{H}$ NMR (400 MHz, $\mathrm{CDCl}_{3}$ ) $\delta 0.99$ (9H, s), 1.04 $(9 \mathrm{H}, \mathrm{s}), 1.33(3 \mathrm{H}, \mathrm{s}), 1.37(3 \mathrm{H}, \mathrm{s}), 1.39(1 \mathrm{H}, \mathrm{t}, J=11.2 \mathrm{~Hz}), 1.54(2 \mathrm{H}, \mathrm{m}), 1.55(1 \mathrm{H}, \mathrm{q}, J=11.2 \mathrm{~Hz}), 1.68(2 \mathrm{H}, \mathrm{m}), 1.78$ $(2 \mathrm{H}, \mathrm{dd}, J=6.8,2.4 \mathrm{~Hz}), 1.88(1 \mathrm{H}, \mathrm{q}, J=11.7 \mathrm{~Hz}), 2.04(1 \mathrm{H}, \mathrm{ddd}, J=11.7,3.4,3.4 \mathrm{~Hz}), 2.09(1 \mathrm{H}, \mathrm{br} \mathrm{s}, \mathrm{OH}), 2.13(1 \mathrm{H}$, dd, $J=11.7,4.9 \mathrm{~Hz}), 2.47(1 \mathrm{H}, \mathrm{ddd}, J=11.7,3.9,3.9 \mathrm{~Hz}), 2.61(1 \mathrm{H}, \mathrm{s}, \mathrm{OH}), 3.12(1 \mathrm{H}, \mathrm{ddd}, J=11.7,9.3,3.9 \mathrm{~Hz}), 3.17$ 
(1H, dd, $J=11.7,3.4 \mathrm{~Hz}), 3.29(1 \mathrm{H}, \mathrm{ddd}, J=11.7,9.8,4.9 \mathrm{~Hz}), 3.33(1 \mathrm{H}, \mathrm{ddd}, J=10.2,10.2,4.9 \mathrm{~Hz}), 3.63(2 \mathrm{H}, \mathrm{m})$, $3.72(1 \mathrm{H}, \mathrm{t}, J=2.4 \mathrm{~Hz}), 3.79(1 \mathrm{H}, \mathrm{t}, J=10.3 \mathrm{~Hz}), 3.81(3 \mathrm{H}, \mathrm{m}), 4.14(1 \mathrm{H}, \mathrm{dd}, J=10.3,4.9 \mathrm{~Hz}):{ }^{13} \mathrm{C} \mathrm{NMR}(100 \mathrm{MHz}$, $\left.\mathrm{CDCl}_{3}\right) \delta 19.9,20.3,21.3,22.6,26.9,27.1(3 \mathrm{xC}), 27.4(3 \mathrm{xC}), 29.2,32.3,35.5,38.4,43.4,62.7,66.8,70.7,72.8,73.9$, 75.0, 75.1, 75.8, 77.2, 78.1, 79.0, 82.7; FABMS m/z $543\left(\mathrm{MH}^{+}\right)$; HRFABS $m / z$ calcd for $\mathrm{C}_{28} \mathrm{H}_{51} \mathrm{O}_{8} \mathrm{Si}_{\left(\mathrm{MH}^{+}\right)}$543.3353, found 543.3327. Anal. Calcd for $\mathrm{C}_{28} \mathrm{H}_{50} \mathrm{O}_{8} \mathrm{Si}$ : C, 61.95; H, 9.29, found C, 61.63; H, 9.04.

(ii) Benzylation. To a solution of the diol $(54 \mathrm{mg}, 0.100 \mathrm{mmol})$ in DMF $(2.0 \mathrm{~mL})$ at $0{ }^{\circ} \mathrm{C}$ were added benzyl bromide $(0.120 \mathrm{~mL}, 1.00 \mathrm{mmol})$ and $\mathrm{NaH}(60 \%$ in mineral oil, $100 \mathrm{mg}, 2.51 \mathrm{mmol})$, and the reaction mixture was stirred at $0{ }^{\circ} \mathrm{C}$ for $15 \mathrm{~min}$ and at room temperature for $2 \mathrm{~h}$. The reaction mixture was cooled to $0{ }^{\circ} \mathrm{C}$, quenched with saturated aqueous $\mathrm{NH}_{4} \mathrm{Cl}$, and extracted with EtOAc. The extract was washed with water and brine, dried, and concentrated in vacuo. Purification by flash chromatography (15\% EtOAc in hexane) gave dibenzyl ether (62 mg, 86\%) as a solid. Mp $130-131{ }^{\circ} \mathrm{C} ;[\alpha]_{\mathrm{D}}^{25}-13.96\left(c 0.62, \mathrm{CHCl}_{3}\right)$; IR $\left(\mathrm{CHCl}_{3}\right) 1473,1455,1365,1089,1029,826 \mathrm{~cm}^{-1} ;{ }^{1} \mathrm{H} \mathrm{NMR}(400 \mathrm{MHz}$, $\left.\mathrm{CDCl}_{3}\right) \delta 0.99(9 \mathrm{H}, \mathrm{s}), 1.04(9 \mathrm{H}, \mathrm{s}), 1.32(3 \mathrm{H}, \mathrm{s}), 1.37(3 \mathrm{H}, \mathrm{s}), 1.46(1 \mathrm{H}, \mathrm{t}, J=11.7 \mathrm{~Hz}), 1.49-1.58(3 \mathrm{H}, \mathrm{m}), 1.65(1 \mathrm{H}$, m), 1.69-1.82 (3H, m), $1.85(1 \mathrm{H}, \mathrm{q}, J=11.7 \mathrm{~Hz}), 2.01(1 \mathrm{H}, \mathrm{ddd}, J=11.7,3.4,3.4 \mathrm{~Hz}), 2.17(1 \mathrm{H}, \mathrm{dd}, J=11.7,4.9 \mathrm{~Hz})$, $2.47(1 \mathrm{H}, \mathrm{ddd}, J=11.2,3.9,3.9 \mathrm{~Hz}), 3.13(1 \mathrm{H}, \mathrm{ddd}, J=11.7,9.3,3.9 \mathrm{~Hz}), 3.25(1 \mathrm{H}, \mathrm{dd}, J=11.7,3.4 \mathrm{~Hz}), 3.30(1 \mathrm{H}, \mathrm{m})$, $3.34(1 \mathrm{H}, \mathrm{ddd}, J=10.3,9.3,4.9 \mathrm{~Hz}), 3.46(2 \mathrm{H}, \mathrm{m}), 3.52(1 \mathrm{H}, \mathrm{t}, J=2.4 \mathrm{~Hz}), 3.80(1 \mathrm{H}, \mathrm{t}, J=10.3 \mathrm{~Hz}), 3.81(1 \mathrm{H}, \mathrm{m})$, $3.84(1 \mathrm{H}, \mathrm{ddd}, J=10.3,9.3,4.4 \mathrm{~Hz}), 3.95(1 \mathrm{H}, \mathrm{dd}, J=11.7,3.9 \mathrm{~Hz}), 4.14(1 \mathrm{H}, \mathrm{dd}, J=10.3,4.9 \mathrm{~Hz}), 4.49(2 \mathrm{H}, \mathrm{s}), 4.57$ and 4.87 (each $1 \mathrm{H}, \mathrm{d}, J=12.5 \mathrm{~Hz}), 7.18-7.36(10 \mathrm{H}, \mathrm{m}):{ }^{13} \mathrm{C} \mathrm{NMR}\left(100 \mathrm{MHz}, \mathrm{CDCl}_{3}\right) \delta 19.9,21.3,21.6,22.6,26.0$, 27.1 (3xC), 27.2, 27.4 (3xC), 32.3, 36.6, 38.5, 43.6, 66.8, 70.2, 72.8, 72.9, 73.9, 74.4, 74.5, 74.7, 77.49, 77.54, 78.1, 78.9, 78.9, 82.6, 127.0 (2xC), 127.2, 127.5 (2xC), 127.6, 128.2 (2xC), 128.3 (2xC), 138.6, 139.7; FABMS m/z 723 $\left(\mathrm{MH}^{+}\right)$; HRFABMS $m / z$ calcd for $\mathrm{C}_{42} \mathrm{H}_{63} \mathrm{O}_{8} \mathrm{Si}\left(\mathrm{MH}^{+}\right)$723.4292, found 723.4278. Anal. Calcd for $\mathrm{C}_{42} \mathrm{H}_{62} \mathrm{O}_{8} \mathrm{Si}: \mathrm{C}, 69.76 ; \mathrm{H}$, 8.65, found C, 69.98; H, 8.83.

(iii) Desilylation. To a solution of the dibenzyl ether $(75 \mathrm{mg}, 0.104 \mathrm{mmol})$ in THF (1.0 mL) were added AcOH (0.018 $\mathrm{mL}, 0.311 \mathrm{mmol})$ and $\mathrm{Bu}_{4} \mathrm{NF}(0.311 \mathrm{~mL}$ of a $1.0 \mathrm{M}$ solution in THF, $0.311 \mathrm{mmol})$, and the reaction mixture was stirred at room temperature for $1.5 \mathrm{~h}$. The reaction mixture was concentrated in vacuo and the residue was purified by flash chromatography (EtOAc) to give diol $22(59 \mathrm{mg}, 98 \%)$ as a solid. Mp 89-91 ${ }^{\circ} \mathrm{C}$; $[\alpha]_{\mathrm{D}}^{25}+6.28\left(c 1.0, \mathrm{CHCl}_{3}\right)$; IR $\left(\mathrm{CHCl}_{3}\right) 3600,3451,1455,1380,1361,1087,1026 \mathrm{~cm}^{-1} ;{ }^{1} \mathrm{H}$ NMR $\left(400 \mathrm{MHz}, \mathrm{CDCl}_{3}\right) \delta 1.32(3 \mathrm{H}, \mathrm{s}), 1.36(3 \mathrm{H}, \mathrm{s}), 1.47$ $(1 \mathrm{H}, \mathrm{t}, J=11.2 \mathrm{~Hz}), 1.51(2 \mathrm{H}, \mathrm{m}), 1.55(1 \mathrm{H}, \mathrm{q}, J=11.7 \mathrm{~Hz}), 1.64(1 \mathrm{H}, \mathrm{m}), 1.69-1.81(3 \mathrm{H}, \mathrm{m}), 1.85(1 \mathrm{H}, \mathrm{q}, J=11.7$ Hz), $2.01(1 \mathrm{H}, \mathrm{ddd}, J=11.7,3.4,3.4 \mathrm{~Hz}), 2.18(1 \mathrm{H}, \mathrm{dd}, J=11.2,4.9 \mathrm{~Hz}), 2.20(2 \mathrm{H}, \mathrm{br}, \mathrm{OHx} 2), 2.44(1 \mathrm{H}, \mathrm{ddd}, J=11.7$, 3.9, $3.9 \mathrm{~Hz}), 3.09(1 \mathrm{H}, \mathrm{ddd}, J=11.7,9.3,3.9 \mathrm{~Hz}), 3.21-3.31(3 \mathrm{H}, \mathrm{m}), 3.47(2 \mathrm{H}, \mathrm{m}), 3.53(1 \mathrm{H}, \mathrm{t}, J=2.9 \mathrm{~Hz}), 3.71(1 \mathrm{H}$, ddd, $J=10.7,9.3,4.9 \mathrm{~Hz}), 3.77(1 \mathrm{H}, \mathrm{dd}, J=11.2,4.9 \mathrm{~Hz}), 3.78(1 \mathrm{H}, \mathrm{m}), 3.85(1 \mathrm{H}, \mathrm{dd}, J=11.2,3.9 \mathrm{~Hz}), 3.95(1 \mathrm{H}, \mathrm{dd}$, $J=11.7,3.9 \mathrm{~Hz}), 4.49(2 \mathrm{H}, \mathrm{s}), 4.58$ and 4.88 (each $1 \mathrm{H}, \mathrm{d}, J=12.7 \mathrm{~Hz}), 7.24-7.36(10 \mathrm{H}, \mathrm{m}):{ }^{13} \mathrm{C} \mathrm{NMR}(100 \mathrm{MHz}$, $\left.\mathrm{CDCl}_{3}\right) \delta 21.3,21.6,25.9,27.2,32.3,36.6,38.4,43.6,63.1,67.0,70.2,72.9,73.8,74.4,74.7,76.7,77.2,77.5,78.5$, 78.8, 81.7, 82.4, 127.0 (2xC), $127.2(2 \times C), 127.5,127.6(2 \times C), 128.2(2 \times C), 128.3,138.5,139.7$; FABMS m/z 583 $\left(\mathrm{MH}^{+}\right)$; HRFABMS $\mathrm{m} / \mathrm{z}$ calcd for $\mathrm{C}_{34} \mathrm{H}_{47} \mathrm{O}_{8}\left(\mathrm{MH}^{+}\right)$583.3268, found 583.3279. Anal. Calcd for $\mathrm{C}_{34} \mathrm{H}_{46} \mathrm{O}_{8}: \mathrm{C}, 70.06$; $\mathrm{H}$, 7.96, found $\mathrm{C}, 70.42 ; \mathrm{H}, 8.23$. 
Compound 5
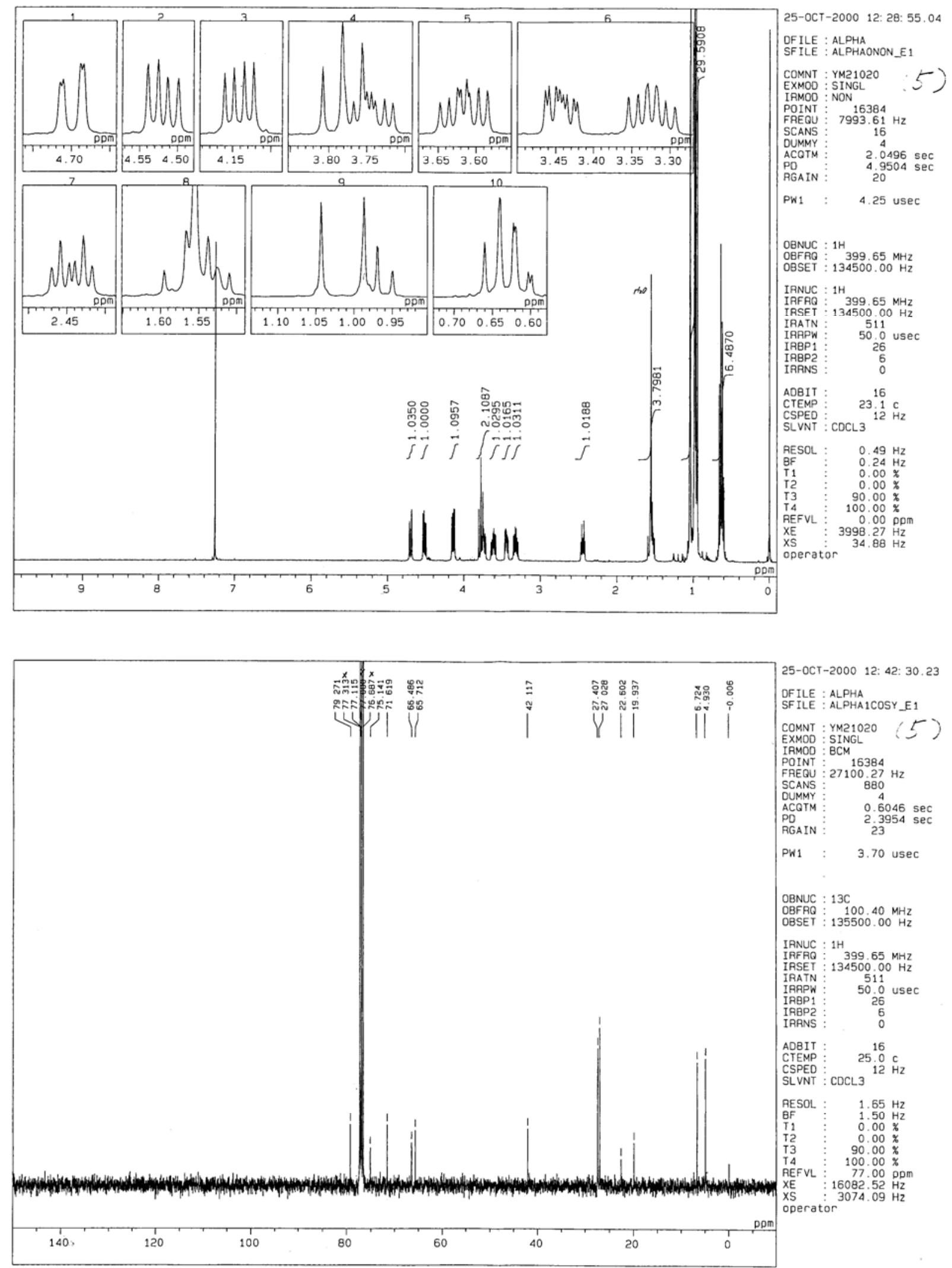

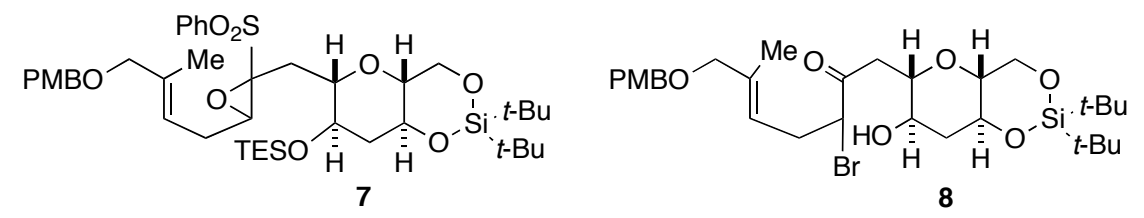

\section{Compound 7}

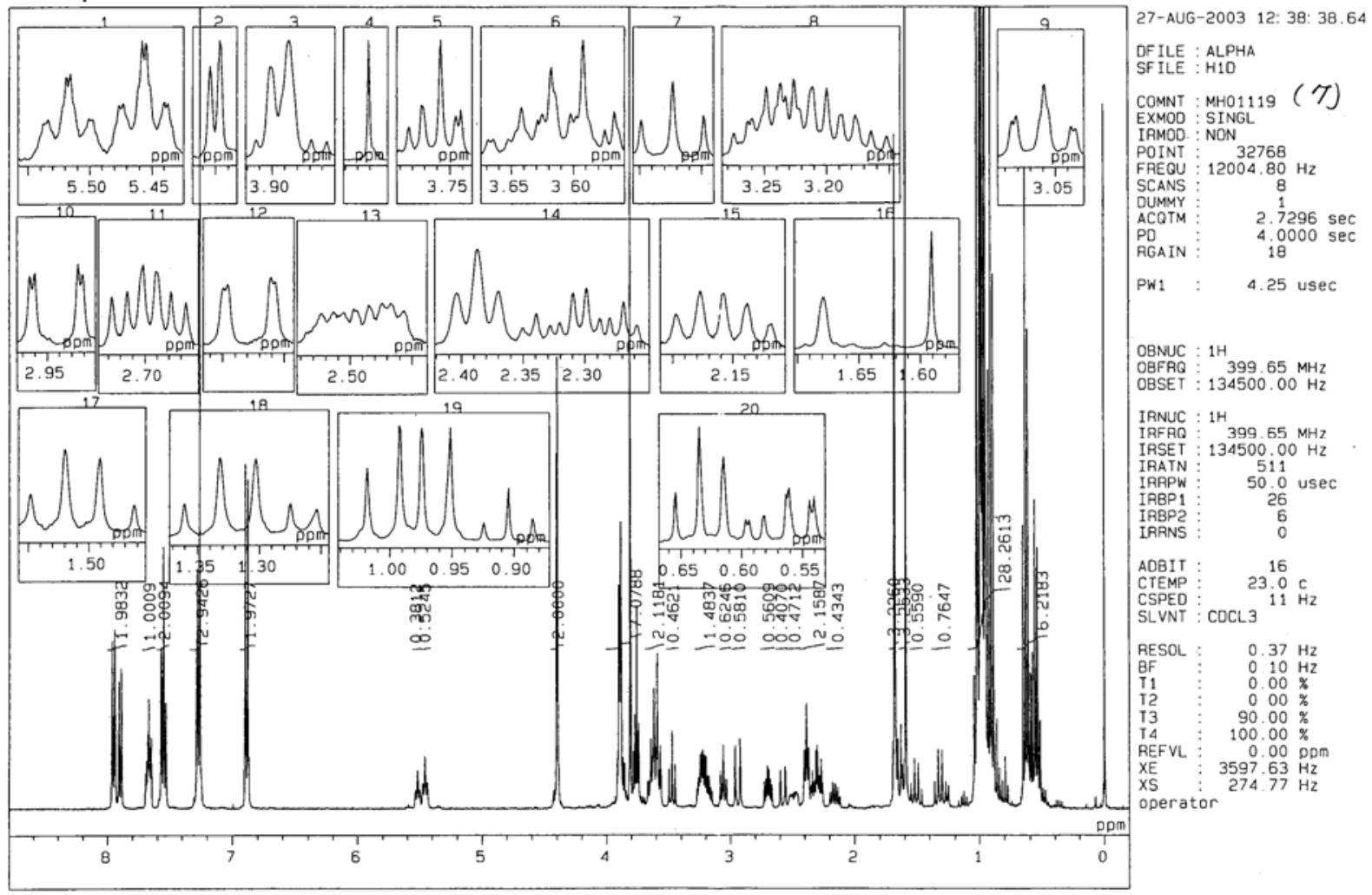

Compound 8

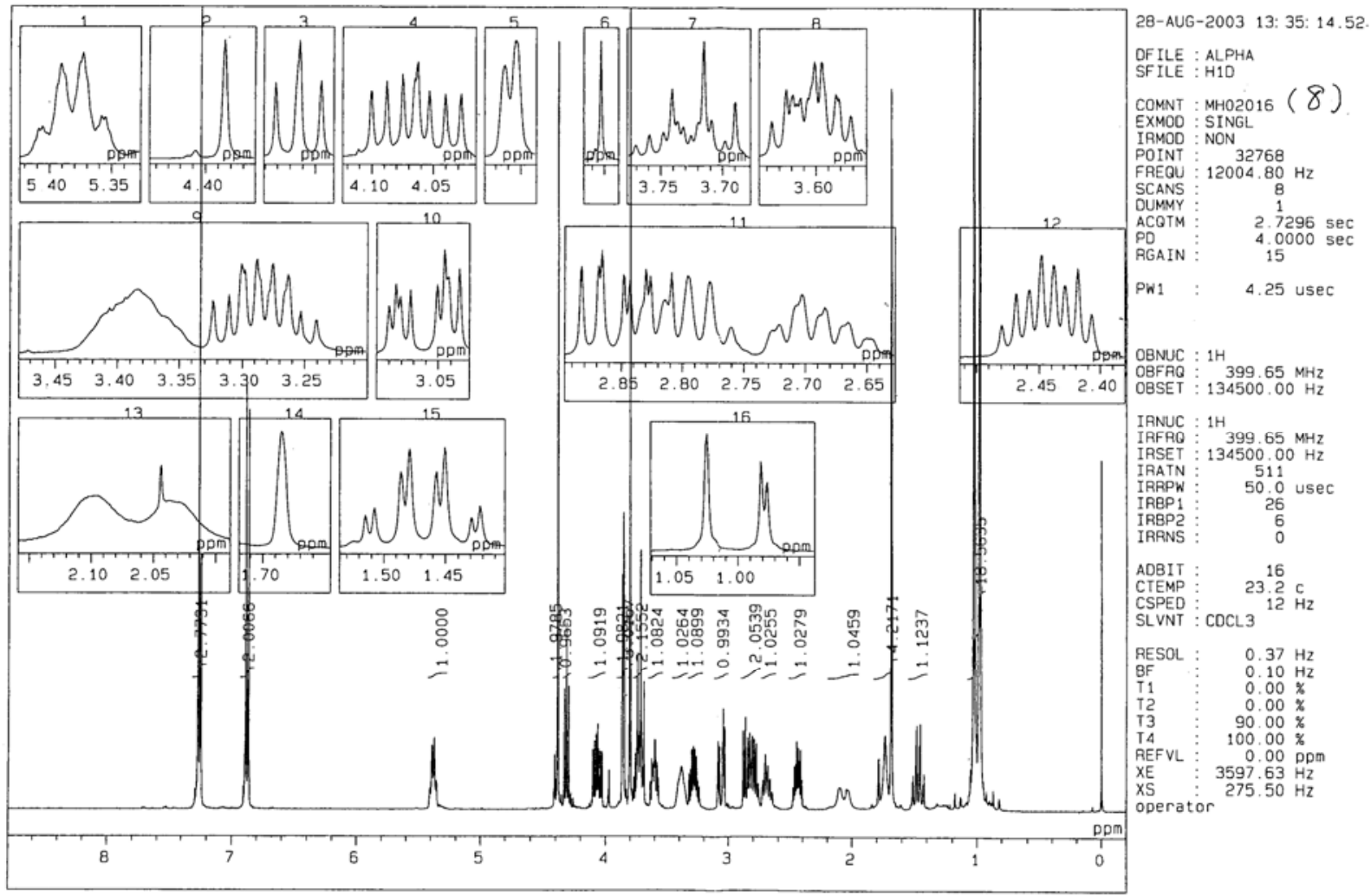




\section{Compound 9}
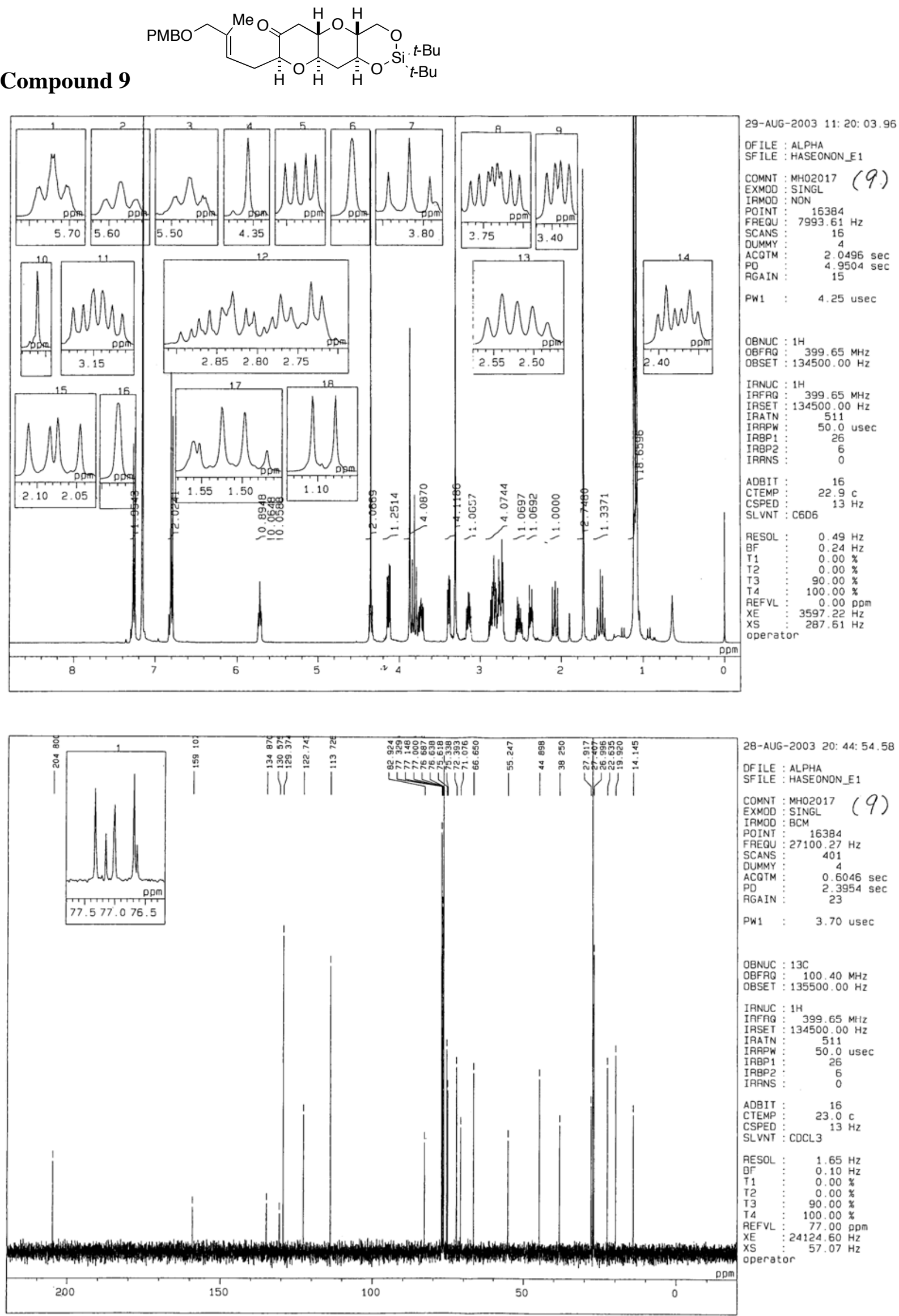
Compound 10
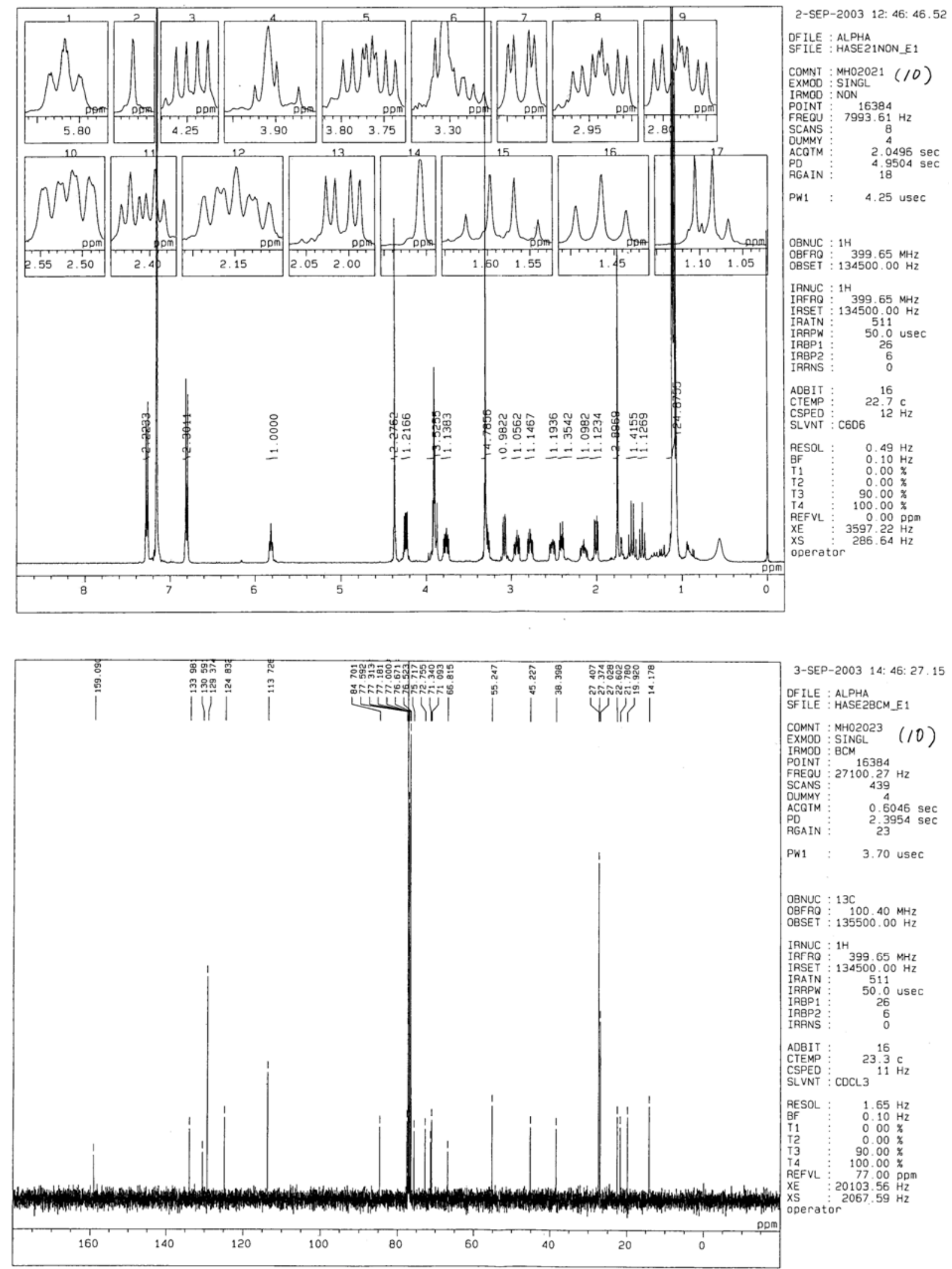


\section{Compound 11}
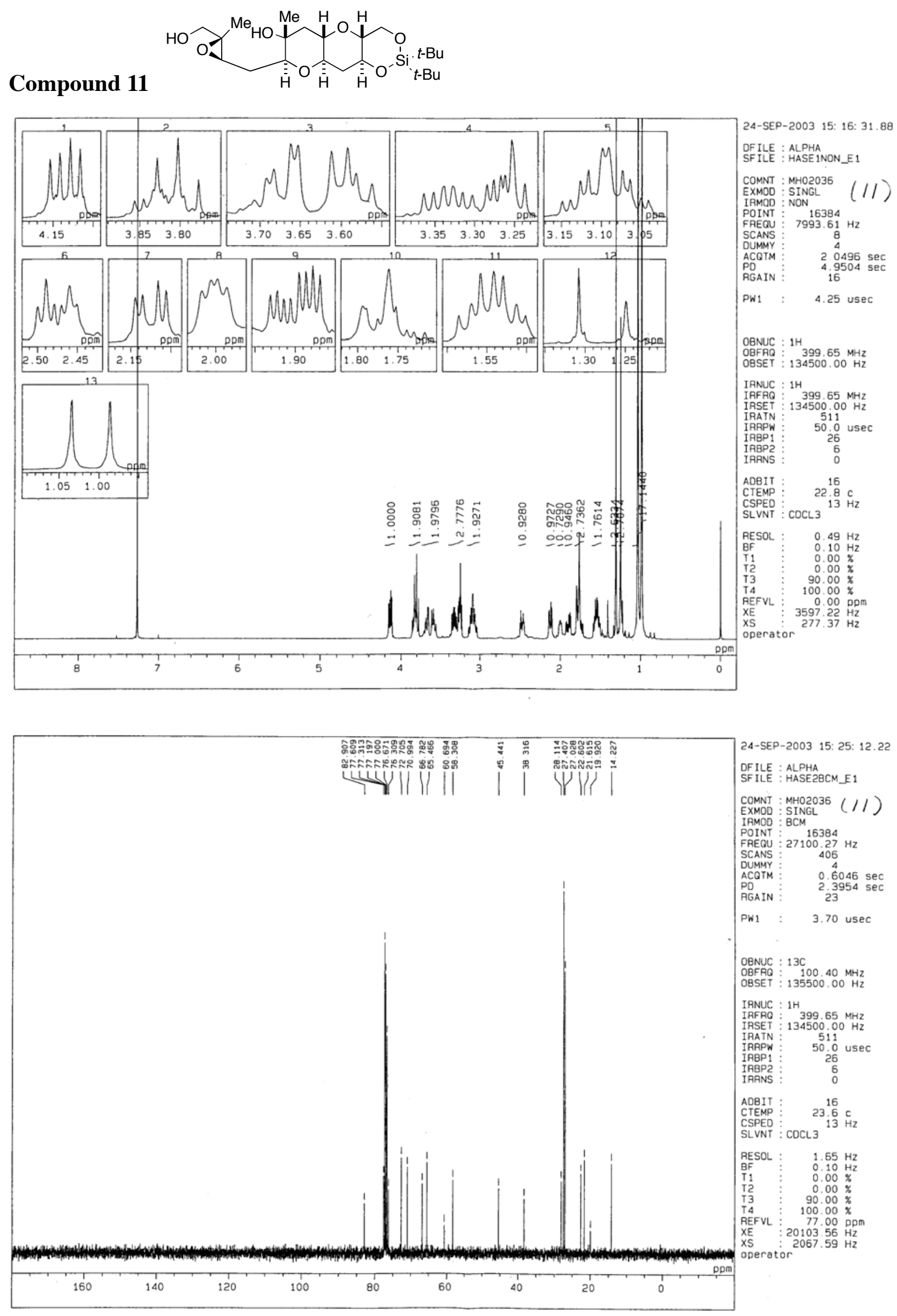
Compound 12

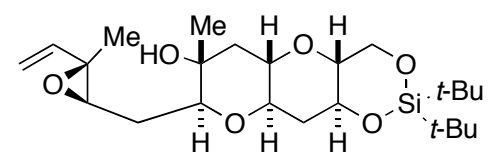
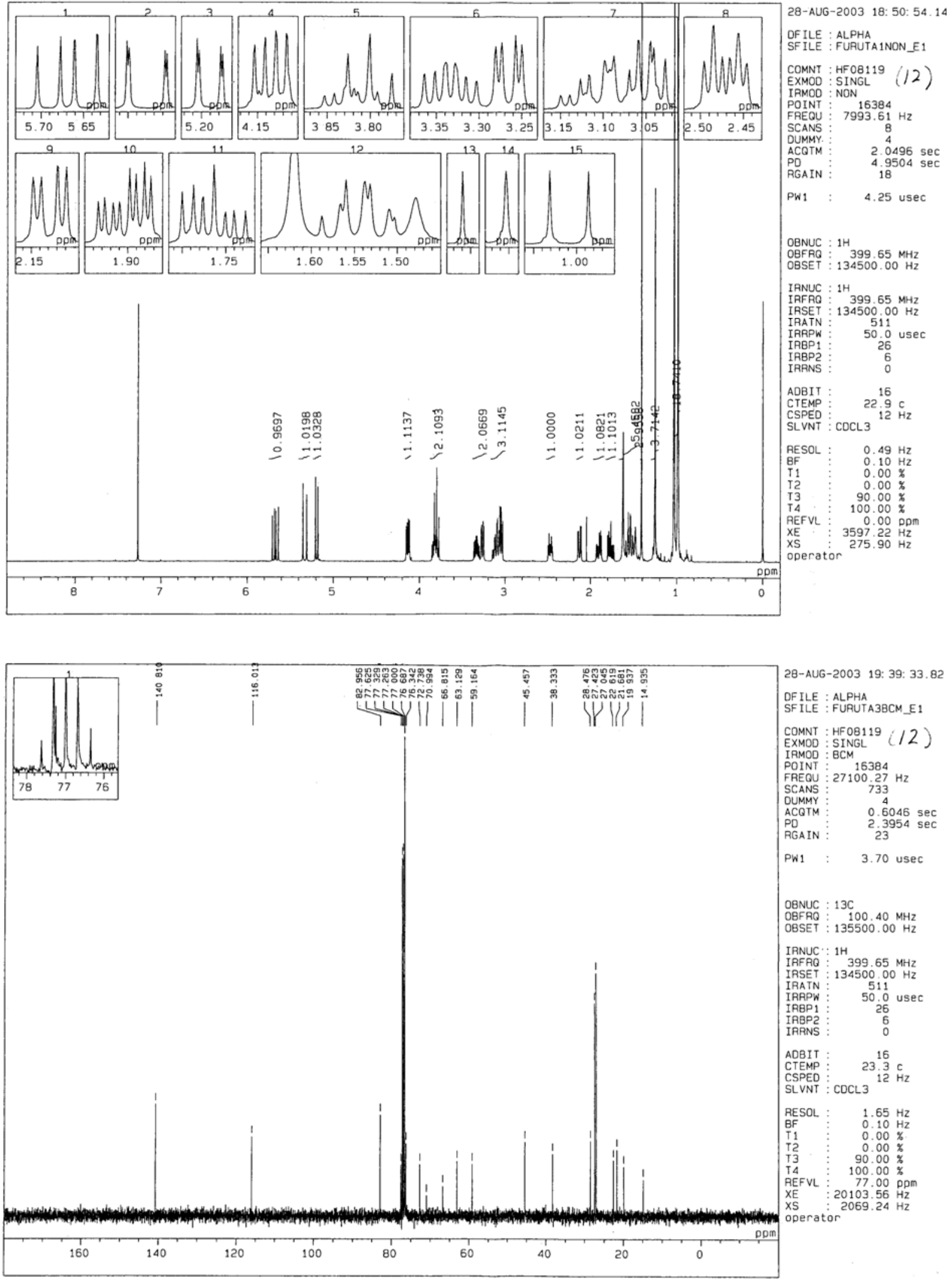


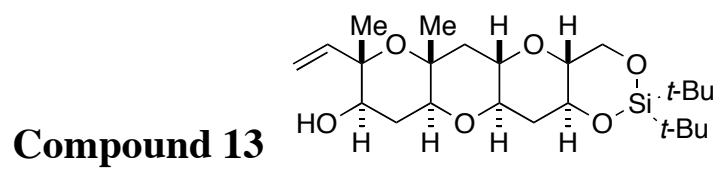
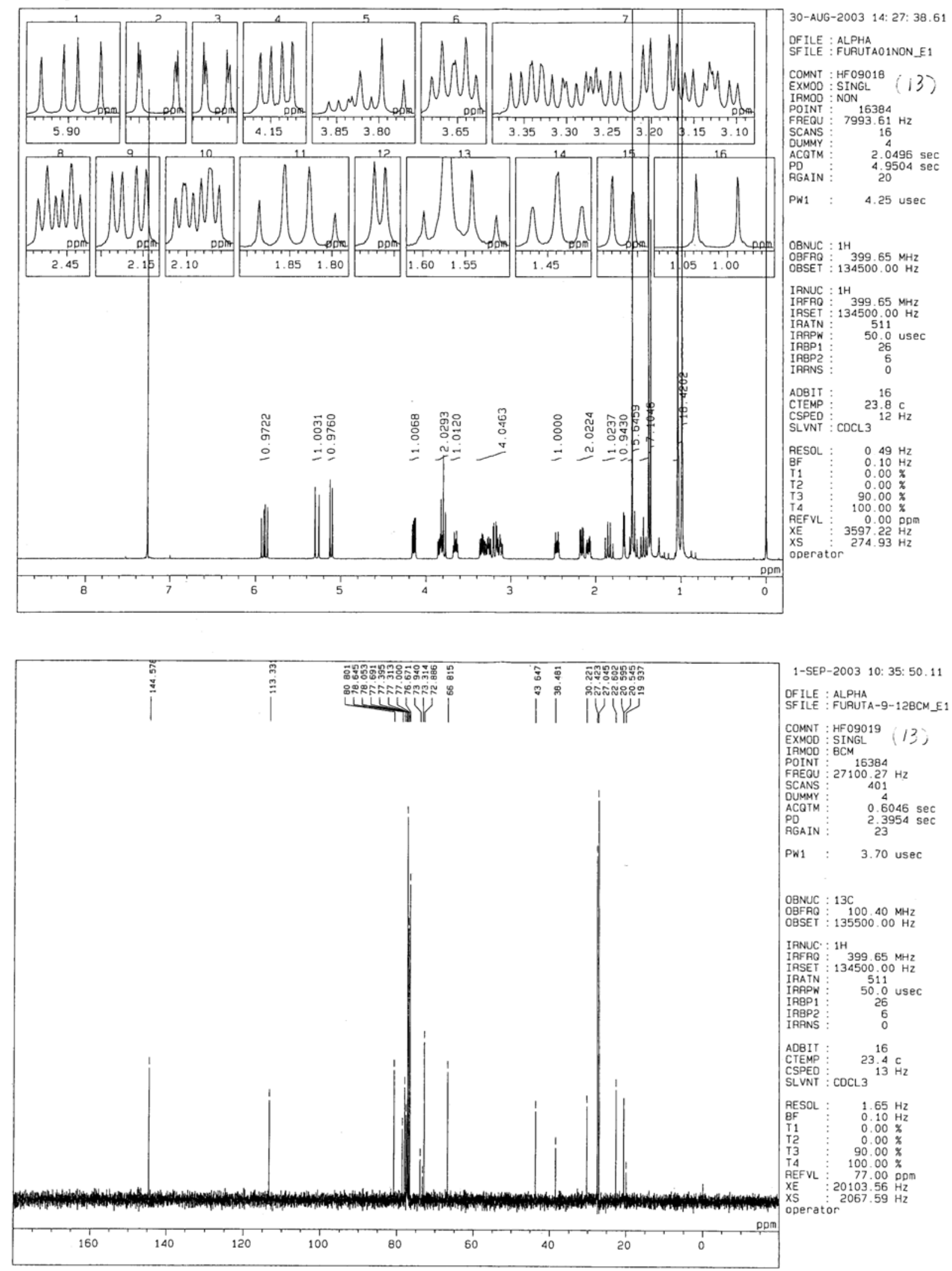
Compound $14 \overbrace{\overline{\mathrm{H}}}^{{ }_{\mathrm{H}}}$
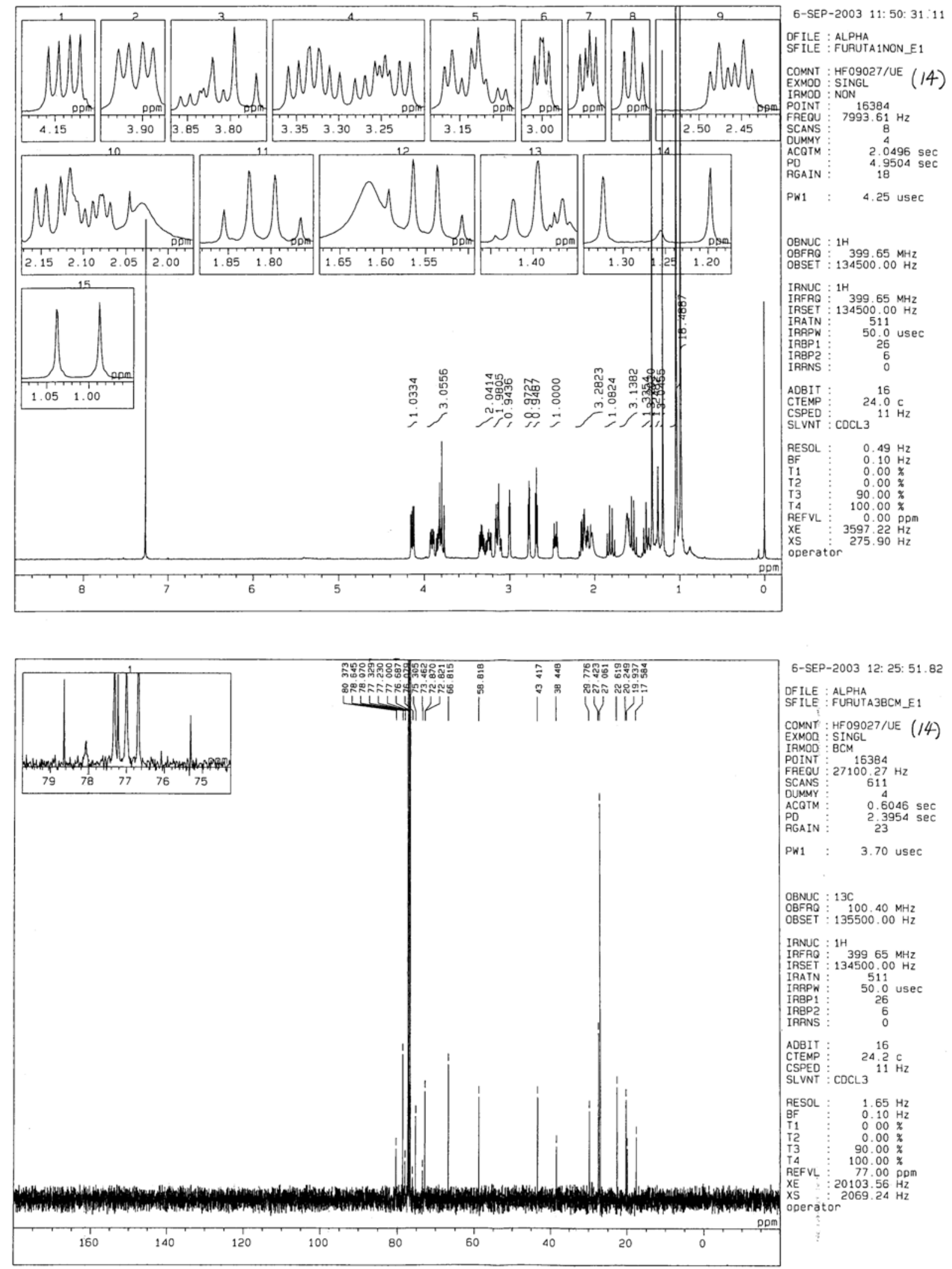
Compound 15
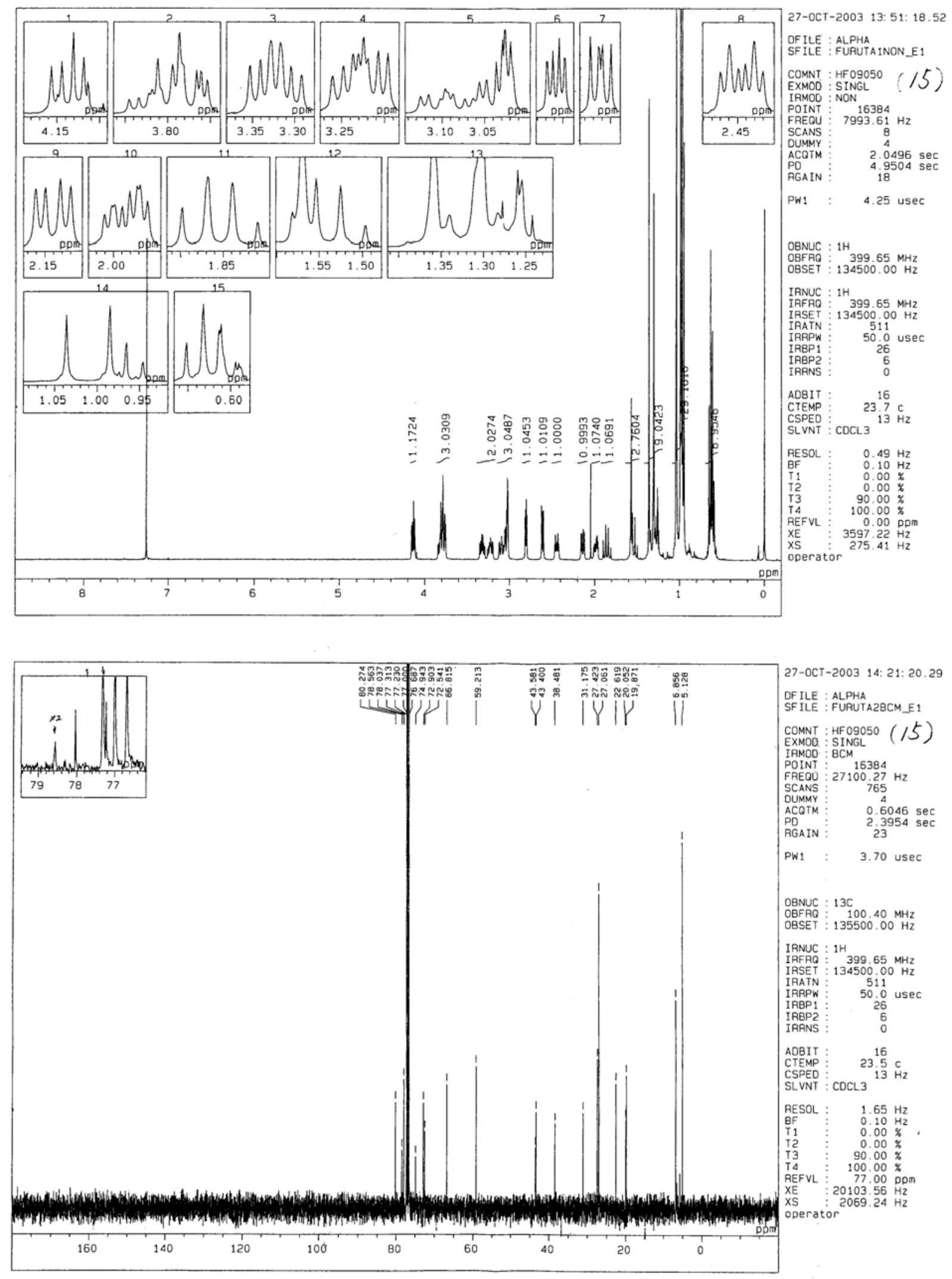

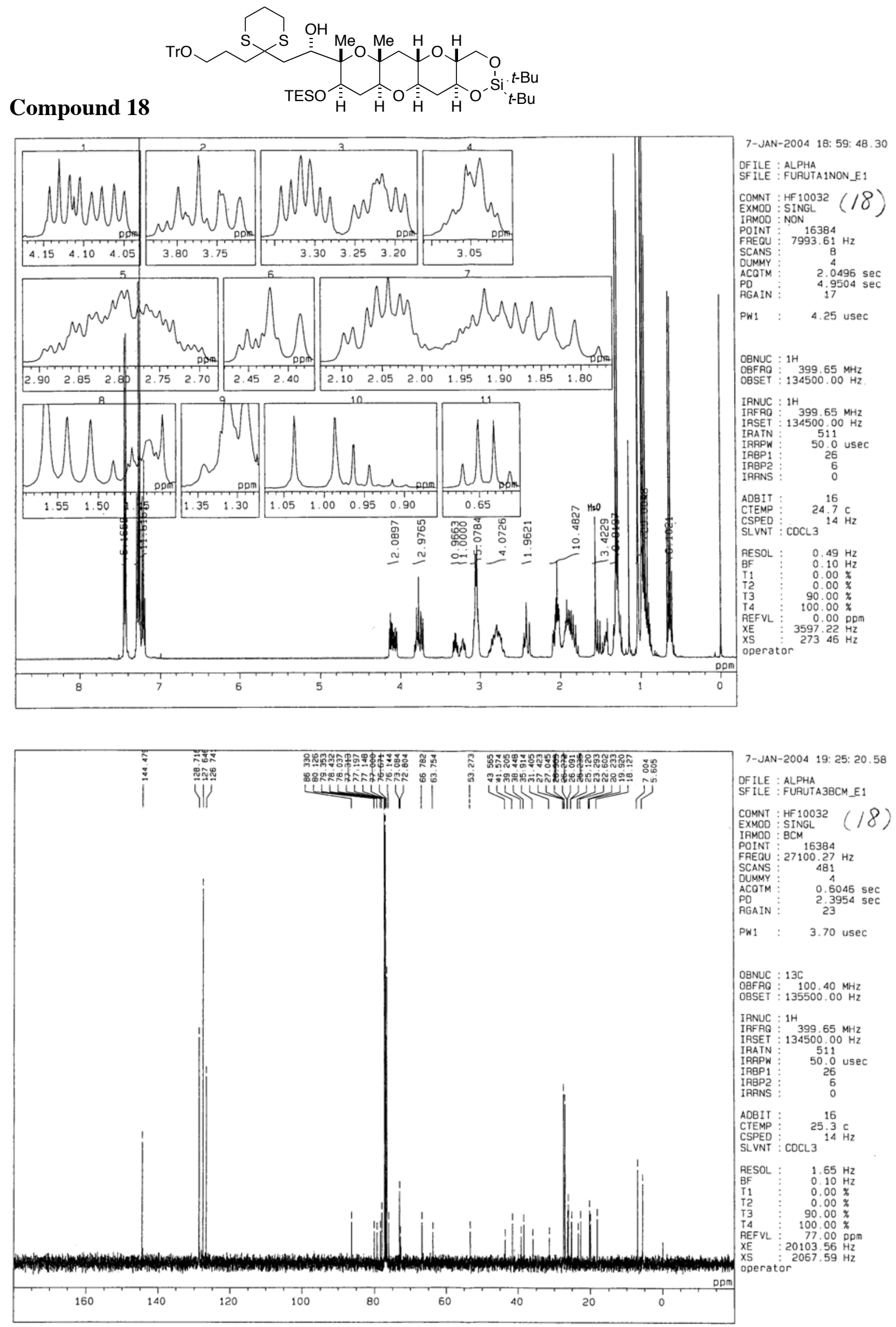
Compound 19 (1)
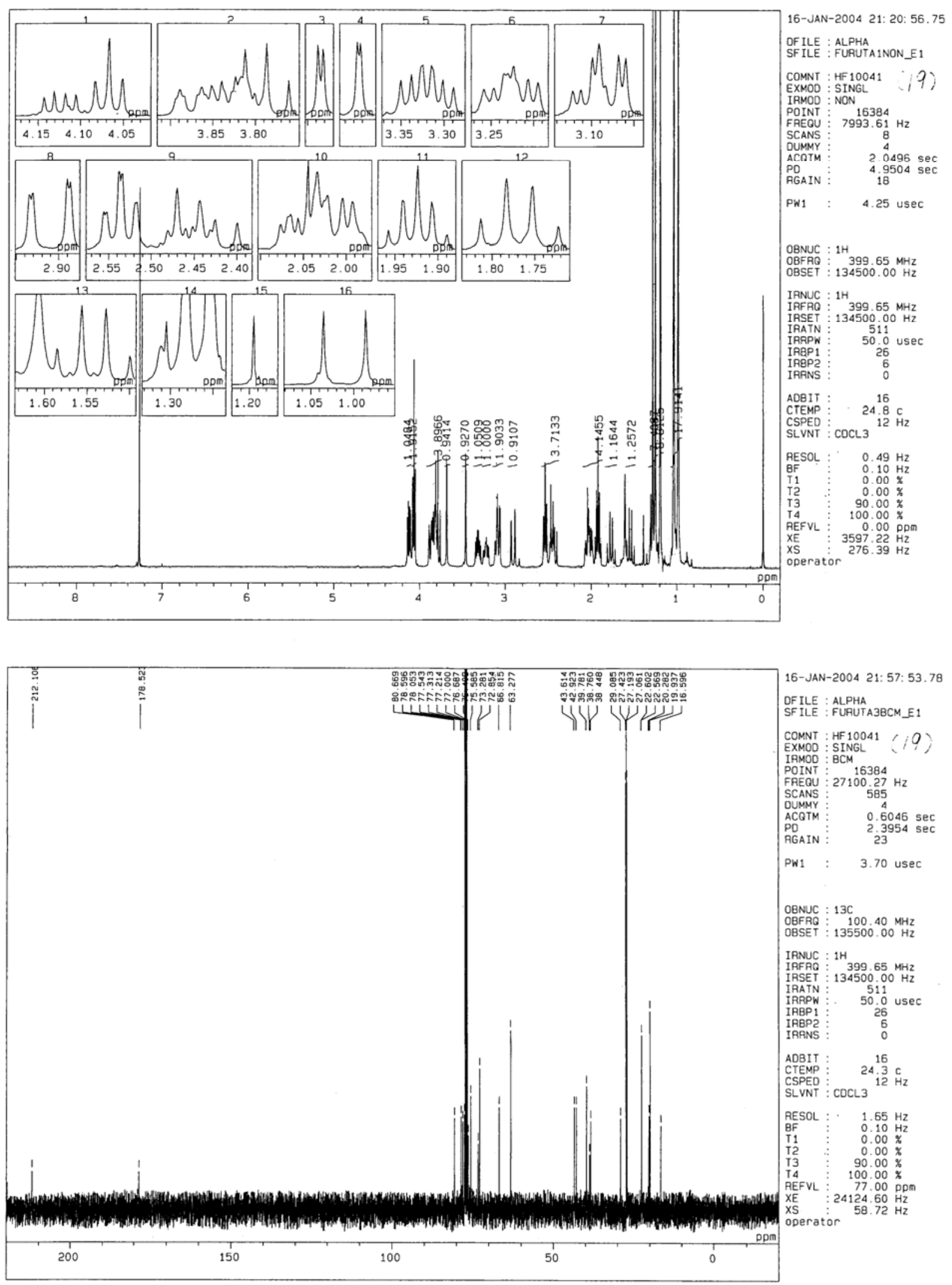

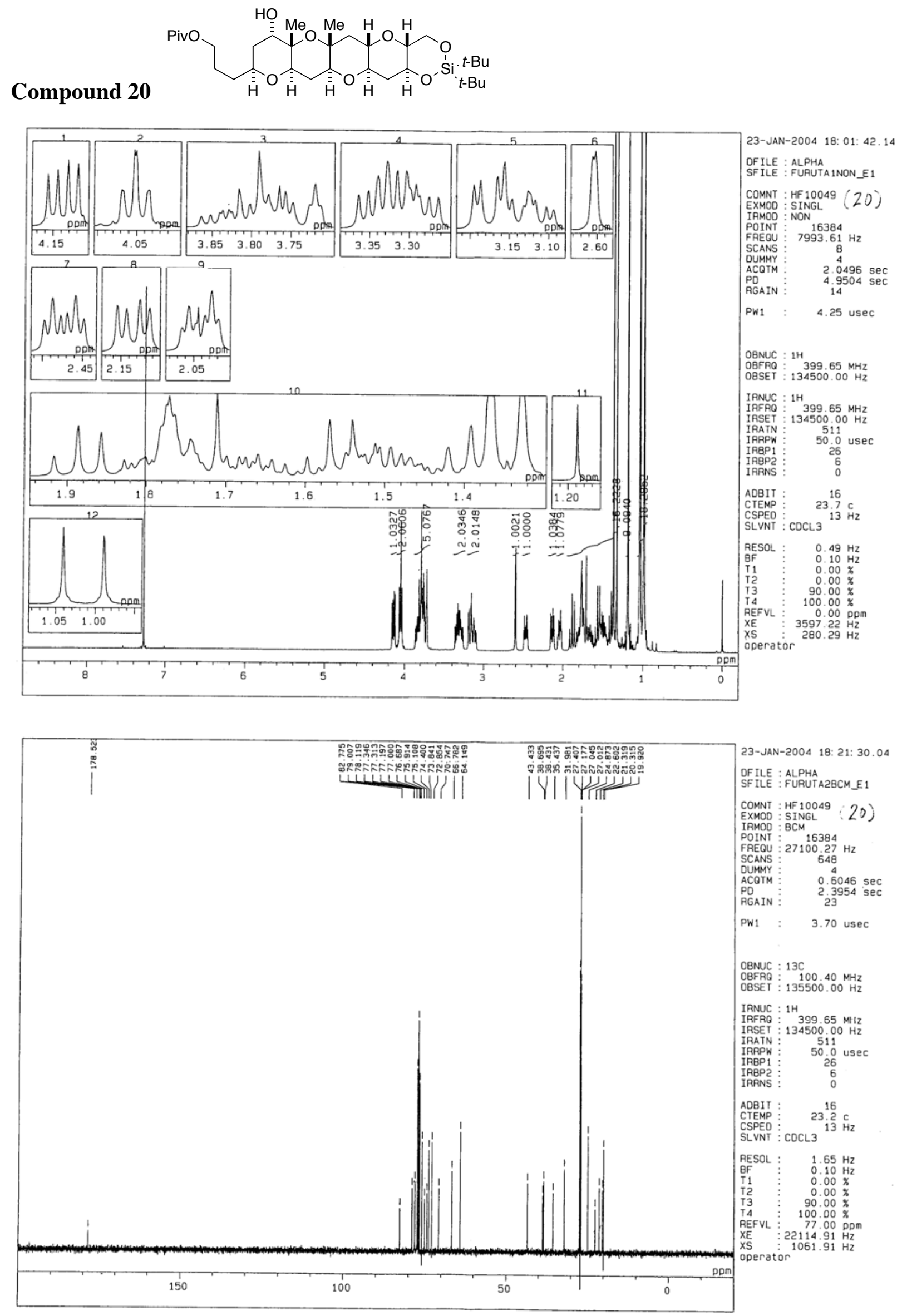

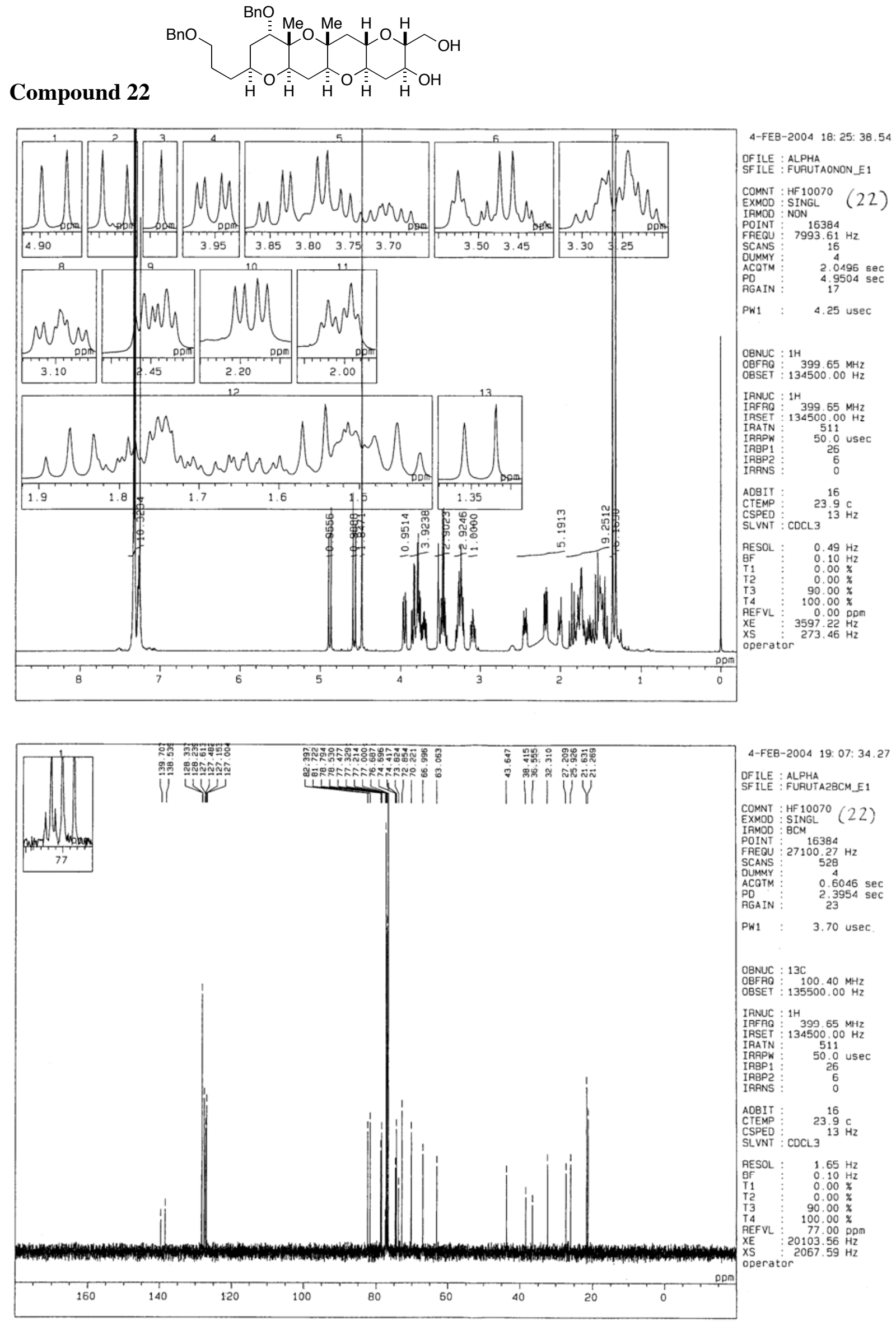